\title{
Dynamics of Meiotic Sex Chromosome Inactivation and Pachytene Activation in Mice Spermatogenesis
}

Ábel Vértesy ${ }^{1,2}$; Javier Frias-Aldeguer ${ }^{1,4}$; Zeliha Sahin ${ }^{1,3}$; Nicolas Rivron ${ }^{1,4}$; Alexander van Oudenaarden $^{1,2}$ and Niels Geijsen ${ }^{1,5}$

1. Hubrecht Institute-KNAW (Royal Netherlands Academy of Arts and Sciences) and University Medical Center, 3584 CT Utrecht, The Netherlands

2. Department of Genetics, Center for Molecular Medicine, Cancer Genomics Netherlands, University Medical Center Utrecht, The Netherlands

3. Amsterdam UMC, University of Amsterdam, Clinical Genetics, Amsterdam Medical Research, Meibergdreef 9, Amsterdam, Netherlands

4. MERLN Institute for Technology-Inspired Regenerative Medicine, Maastricht University, The Netherlands

5. Faculty of Veterinary Medicine, Clinical Sciences of Companion Animals, Utrecht University, The Netherlands

\section{Abstract}

During germ cell development, cells undergo a drastic switch from mitosis to meiosis to form haploid germ cells. Sequencing and computational technologies now allow studying development at the single-cell level. Here we developed a multiplexed trajectory reconstruction to create a high-resolution developmental map of spermatogonia and prophase-I spermatocytes from testes of a Dazl-GFP reporter mouse. We identified three main transitions in the meiotic prophase-I: meiotic entry, the meiotic sex chromosome inactivation (MSCI), and concomitant pachytene activation. We validated the key features of these transitions in vivo using single molecule FISH. Focusing on $\mathrm{MSCl}$, we found that $34 \%$ of sex chromosomal genes are induced shortly before $\mathrm{MSCl}$, that silencing time is diverse and correlates with specific gene functions. These highlight a previously underappreciated level of regulation of MSCl. Finally, we found that spermatozoal genes in pachytene are activated in a temporal pattern reflecting the future anatomic and functional order of the sperm cell. Altogether we highlighted how precise and sequential changes in gene expression regulate cellular states in meiotic prophase-I. 


\section{Introduction}

Spermatogenesis is a complex and continuous differentiation process from the spermatogonia to the spermatocyte stages that resolves a switch from mitotic to meiotic divisions to generate haploid sperm cells. This process is accompanied by major morphological, cellular and transcriptional changes, which are not well understood transcriptome-wide nor temporally resolved in a fine manner. Single-cell analysis provided an overall description of spermatogenesis (Chen et al., 2018; Jung et al., 2018; Lukassen et al., 2018) including the analysis of Sertoli cell biology (Green et al., 2018), the comparison of human and mouse spermatogenesis (Hermann et al., 2018) and the analysis of post-meiotic sex chromosome dynamics (Ernst et al., 2019). Here, we developed a multiplex computational approach to finely resolve developmental trajectories of single Dazl+ cells over the time of mitotic to meiosis transition.

A male specific feature of meiosis is the incomplete pairing of the $X$ and $Y$ chromosomes during meiosis (termed asynapsis). While in female cells, all chromosomes can form homologous pairs and recombine, in males, the $X$ and $Y$ chromosomes only share a small homologous part, the pseudoautosomal region (PAR). The remaining non-homologous regions stay unpaired, acquire repressive chromatin marks, and eventually form the sexbody within the nucleus (Khalil, Boyar and Driscoll, 2004; van der Heijden et al., 2007). This silencing of the $X$ and $Y$ chromosome during male meiosis is called meiotic sex chromosome inactivation (MSCI) (Henderson, 1963; Turner, 2015). $\mathrm{MSCl}$ is thought as a special case of meiotic silencing of unsynapsed chromatin (Shiu et al., 2001) and is an essential step of male meiosis (Royo et al., 2015). While MSCl's post transcriptional regulation, such as $\mathrm{H} 2 \mathrm{AX}$ phosphorylation (Mahadevaiah et al., 2001) and ubiquitination (Baarends et al., 2005) are well described, the transcriptional control of these regulators and the dynamics of gene expression silencing are still to be identified.

Another feature of male meiosis is a massive wave of gene expression at the pachytene stage (Fallahi et al., 2010), when mid-meiosis cells express a remarkable array of postmeiotic genes involved in spermiogenesis (da Cruz et al., 2016). The dynamics of the activation of this transcriptional program, and whether sex chromosomal genes can escape $\mathrm{MSCl}$ are open questions in the field, which we analyzed in detail.

We thus set out to draw a temporally resolved, transcriptome-wide gene expression atlas from meiotic commitment until prophase-I, in order to unravel transcriptional changes that coordinate germ cell differentiation and meiotic transition. We sequenced mRNA from 
1880 single cells, multiplex reconstructed their temporal order in silico, which we validated via microscopy. This allowed us to describe the precise sequence of transcriptome-wide changes upon $\mathrm{MSCl}$ and pachytene activation.

\section{Results}

1. Single-cell transcriptomics of Dazl+ germ cells identifies stem cells along with 4 stages of prophase-I

Dazl is expressed from spermatogonial to early pachytene stage (Kim, Cooke and Rhee, 2012). We thus used Dazl-GFP reporter mice (Chen et al., 2014) to isolate cells during meiotic entry and prophase from testes of adult mice (2-4 month) (Fig. 1A). We applied single-cell mRNA sequencing using the SORT-seq protocol (Muraro et al., 2016), and sequenced 1880 single cells from 3 mice. After trimming and sequence alignment to the mm10 reference transcriptome (Online Methods), we filtered low quality cells with fewer than 3000 unique (UMI corrected) transcripts and selected 1274 high-quality germ cells. These were normalized to the median transcript count (20488 per cell), allowing us to compare the relative expression across cells (Fig. S1A, Online Methods).

To determine whether transcriptional changes are continuous or rather separated into discrete steps, we classified cells by transcriptome-wide, unsupervised k-medoids clustering on Spearman correlation coefficients. Cells were clustered in 5 groups (Fig. 1 B, C) based on all genes with at least 10 transcripts in minimum 50 cells. Importantly, we did not predefine the number of expected stages, but estimated it based on the withincluster-dispersion of data points (Grün et al., 2016). The established clusters are also supported by saturating gap statistics, a positive cluster silhouette. High Jaccard indices across bootstrapping rounds indicate that the clusters are reproducible (Fig. S1 C-E, Online Methods). These clusters are not influenced by animal-to-animal variation, batch variation nor library complexity (Fig. S2). After we ruled out batch effects, we excluded contamination from somatic cells based on a lack of expression of Sertoli or Leydig cell specific markers Stra6 and Amh (Fig. 1D) (O'Shaughnessy, Morris and Baker, 2008; Griswold, 2016). On the contrary, cells showed a high expression of Dazl, and of the meiotic prophase marker Sycp3 (Fig. 1D).

Cells forming cluster 1 express markers of differentiating spermatogonia, like Dmrt1 (Matson et al., 2010), Kit (Yoshinaga et al., 1991), and earlier progenitor markers, as 
Lin28a (Chakraborty et al., 2014) (Fig. 1 D). Cells forming the cluster 2 uniquely express Stra8, the key gene marking meiotic entry (Anderson et al., 2008). Cells forming cluster 3 specifically upregulate the zygotene specific Cyclin B3 (Ccnb3) (Nguyen et al., 2002), which is located on the $X$ chromosome, and marks the last stage with sex chromosomal expression (zygotene I). Cells forming cluster 4 upregulated H2A histone family member $X(H 2 a f x)$, the histone variant associated with meiotic sex chromosome inactivation (MSCl) in early pachytene. Finally, cells forming cluster 5 specifically express the pachytene marker lactate dehydrogenase $\mathrm{C}(L d h c)$, a testis-specific enzyme in anaerobic glycolysis (Goldberg et al., 2010). Taken together, we concluded that Dazl+ cells represent 5 transcriptionally distinct subpopulations with clear transcriptional signatures.

\section{Identified clusters correspond to subsequent stages of prophase-I}

To better characterize the established clusters, we compared their gene expression by differential gene expression analysis (Table S1-S2, Data S2). Cluster 1 on the left edge of the t-SNE map (Fig 1B) encloses $\sim 33 \%$ of the cells (Fig. S2A). They are uniquely expressing Dmrt1 and other markers of mitotically dividing cells including Ccnd1, Cenpa, Mcm2, Ccdc6, Gsr, Ccdc6 and Crabp1 (Data S2, Table S1). While most spermatogonial cells are Kit+, we noticed that some markers of the undifferentiated state, including Uch/1 or Lin28a (Luo, Megee and Dobrinski, 2009; Griswold, 2016) are partially co-expressed with Kit+ cells (Data S1).

On the right side of the t-SNE map (Fig 1B), 36\% of the cells form Cluster 5 (Fig. S2A). These cells are characterized by a high expression of pachytene markers such as the Adam genes, Ldha, Ldhc, Crisp2, Ybx genes, Meig1 and Piwil1 (Jan et al., 2017) (Data S2), consistent with early expression of post-meiotic genes (da Cruz et al., 2016).

These two opposite clusters ( 1 and 5 ) are defined by many uniquely expressed genes within our dataset (Table S1-S2). Between these, we found 3 intermediate clusters with fewer specific genes. These clusters therefore are largely determined by unique combinations of up- and downregulated genes. For example, we found that Cluster 2 uniquely expresses Stra8 and Prdm9 suggestive of differentiated spermatogonial or preleptotene stage (Sun et al., 2015). At the same time, they also started to upregulate the meiotic Sycp genes (Margolin et al., 2014).

127 The meiotic entry is accompanied by a global transcriptional silencing (Monesi, 1965; 128 Ernst et al., 2019). Indeed, we observed that the meiotic entry (Cluster 2) corresponds to 
a transient drop in raw transcript counts in clusters 2-4 (Fig. 2D), which is not explained by differences in sequencing depth (Fig. S1F). Despite this drastic silencing, each cluster (1 \& 2) showed the upregulation of a specific subset of genes, as compared to the earlier stage (Table S2).

The next two clusters (3 \& 4) displayed the peak expression of Dazl, Sycp1 \& 3. They also a shared expression of zygotene specific genes involved in recombination, such as Hormad1 (Shin et al., 2010) and Meiob (Luo et al., 2013), as well as Elav/3, Gmland Mnd1 (Data S2). A key difference is that while cluster 3 shows high expression for many sex chromosomal genes (e.g. Scm/2, Fmr1), cells in clusters 4 and 5 only express residual amounts of these mRNAs.

Taken together we concluded that clusters 1 to 5 show unique gene expression patterns, which recapitulated established stages of prophase-I. We built an online explorer for all genes (https://vertesy.shinyapps.io/ExpressionBrowser/) and listed all differentially expressed genes (Table S1-2). We also found subpopulations within these clusters, suggesting finer transitions. To analyze these systematically, we extended our computational analysis to a staging-free approach, better reflecting the continuity of spermatogenesis.

\section{Multiplex trajectory reconstruction precisely recapitulates established} meiotic events

Multiple recent approaches successfully reconstructed pseudotemporal trajectories from single-cell transcriptomes using either principal curves (Petropoulos et al., 2016), TSCAN (Ji and Ji, 2016), or Monocle 2 (Qiu et al., 2017). We reconstructed temporal order in our data set by all three methods (Fig. S3). It resulted in a similar, but not identical ordering (min. pairwise $r>0.95$ ) (Fig. 2A, Online Methods). From the three reconstructions we calculated the consensus trajectory reflecting developmental time (Fig. 2B), which allowed us to analyze transcriptome dynamics in a more reliable and precise way than possible before.

First of all, this map recapitulated the previously determined stages, while revealing much finer events, as detailed below (Fig. 2E). We then used in vivo samples to validate this reconstruction, by 3-color single-molecule RNA fluorescence in situ hybridization (smFISH). We selected the (pre)leptotene gene Stra8 (cluster 2) (Anderson et al., 2008), 
the pachytene specific H2afx (cluster 4) and the gene Actb, which is primarily expressed in spermatids (Ventela et al., 2000). The smFISH clearly pinpointed spatially-separated populations. Cells formed a sparse outer layer of Stra8+ (pre)leptotene cells decorating a dense intermediate layer of $H 2 a f x+$ pachytene cells, enclosing an inner ring of Actb+ spermatid cells (Fig. 2C). Additionally, the three key transcriptional events (silencing at meiotic entry, meiotic sex chromosome inactivation and pachytene activation) are correctly recapitulated (Fig. 2D).

We then focused on finer differences. The reconstruction precisely predicted that Lin28a is expressed only in the earliest stage spermatogonia (Chakraborty et al., 2014). It also accurately predicted that Stra8 peaked shortly before Prdm9 (Sun et al., 2015). Remarkably, all other analyzed marker genes also peaked in the expected developmental order (Fig. 2E). Altogether, we concluded that the consensus trajectory faithfully recapitulated the developmental progress of early spermatogenesis, thus, it allows for a reliable, in-depth computational analysis of gene expression patterns.

\section{Temporal expression maps show continuous differentiation from} (pre)leptotene to pachytene

Next, we analyzed all genes' expression profiles along the trajectory. First, we classified gene expression dynamics, and found that most genes showed a single expression peak (Fig. 3A). We then analyzed the co-expression profiles of genes peaking around (pre)leptotene. This showed how the meiotic master regulator Stra8 expression preceded other preleptotene genes. As an example, we highlighted the cascade of Stra8, Tex15 and Dmc1 activation within one stage (Fig. 3B, selected by highest z-score).

To understand this sequential activation deeper, we selected genes with a single expression maximum along the trajectory (Online Methods, Fig. S4). We then ordered these genes by time of peaking (Fig. 3C). First, it showed the three most unique stages in our dataset: spermatogonia, (pre)leptotene and pachytene. By showing the number of genes changing at each stage, it also highlighted the magnitude of changes upon meiotic entry, and pachytene activation. The analysis also suggests that sequential and sharply timed gene activation might be the regulatory clockwork of spermatogenesis, possibly triggering genes with wider expression profiles as seen in (Fig. 3A, B). 
This analysis allowed us to identify genes co-expressed with known marker genes, which in turn can help to characterize their function. As an example, we identified 21 genes ( $r>$ 0.9) with expression patterns similar to H2afx (Fig. 3D). The best correlating genes were Hemt1 (Gml2) and $\mathrm{Gml}$ (Hemt3) (Fig. 3E), a pair of uncharacterized genes, although $\mathrm{Gml}$ was previously identified in prophase spermatocytes (Xue et al., 1999).

To validate this stunning co-expression of Hemt1 and H2afx, we performed smFISH (Fig. $3 F-G)$. This confirmed their co-expression within a spatially-restricted population, which suggests a co-functionality. To help researchers finding other co-expressed genes, we implemented an interactive co-expression browser as part of our online toolbox. Taken together, we created a compendium of gene expression dynamics in meiotic prophase-I and developed interactive tools for further investigation.

\section{Silencing of an $n-M y c$ transcription factor network coincides with global} silencing during meiotic entry

Previous studies elegantly inferred transcription factor binding sites for large clusters of cells (Green et al., 2018) or clusters genes (Jung et al., 2018) during meiotic entry. Building upon this, we aimed at understanding the transcriptional programs behind the global silencing during meiotic entry. Therefore, we compared the transcriptome of spermatogonial and (pre)leptotene cells (Fig. 4A, Table S3). We analyzed these by identifying significantly enriched GO-terms of $>4$-fold differentially expressed genes (Online Methods, Table S3). The GO-terms were then clustered according to semantic similarity (Supek et al., 2011).

Besides the clear and expected switch from mitotic to meiotic program, the transcriptome of (pre)leptotene cells showed strong metabolic silencing (Fig. 4B, C). Metabolic silencing also may explain the drastic decrease of mRNA content in late spermatogonia, which lasts until pachytene (Fig. 2D). Many housekeeping genes were silenced, and their expression generally varied throughout the entire prophase-I (Data S3).

To better understand how meiotic entry is regulated, we focused on transcription factors. As many transcription factors are expressed at the detection limit of single-cell sequencing, they often cannot be reliably identified by differential gene expression analysis. Therefore, we analyzed the transcription factor binding site enrichment in the promoters of differentially expressed genes (Online Methods) (Matys, 2006; PortalesCasamar et al., 2010; Kuleshov et al., 2016). To gain statistical power, we analyzed >2- 
fold enriched genes in either cluster (Fig. 4D, enriched GO-terms recapitulated previous results, see Table S3).

Eight transcription factors showed highly significant (p.adj. <0.01) binding site enrichment in the spermatogonial genes (Fig. 4E). In contrast, no transcription factor was unique to the (pre)leptotene stage, while some (Sp1 and Smad4) showed a highly significant enrichment in both gene sets (Table S3).

We found that six out of eight of the predicted transcription factors from a tightly interconnected hub (Fig. 4F, Online Methods) based on protein interaction network (Szklarczyk et al., 2017). This pinpoints a core regulatory network may regulate the mitotic-meiotic transition. When testing these predictions on our data, we found that most predicted spermatogonia-specific factors ( $n-M y c$, Trp53, Egr1, Smad4) are silenced expectedly. Curiously Sp1 -which cooperates with Smad4 in TGF- $\beta$ signaling- showed the opposite trend (Feng, Lin and Derynck, 2000). Nrf1 (Wang et al., 2017) was expressed continuously, while Myb and Elk1 were not detected (Fig. 4G).

Taken together, explicit silencing -rather than activation- drives the transcriptional switch in meiotic entry. The silencing showed a particularly strong metabolic control. Myc is known to regulate more than $11 \%$ of all genes, in particular metabolic ones (Fernandez, 2003). Hence, the downregulation of the spermatogonia -specific $n-M y c$ variant (BraydichStolle et al., 2007) may explain the global transcriptional silencing upon meiotic entry.

6. $34 \%$ of the sex chromosome genes are induced right before sequential inactivation by $\mathrm{MSCl}$.

245 The second major wave of transcriptional silencing in prophase-I is the meiotic sex chromosome inactivation (MSCl). This leads to the global silencing of the sex chromosomes (Henderson, 1963; McKee and Handel, 1993). While post-meiotic silencing has been analyzed in detail (Ernst et al., 2019), and multiple recent single-cell studies referred to $\mathrm{MSCl}$ (Chen et al., 2018; Jung et al., 2018; Lukassen et al., 2018). However, the patterns of gene silencing during $\mathrm{MSCl}$, and the underlying regulation remained open questions.

To tackle this question, we classified all sex chromosomal genes along the trajectory. This confirmed their silencing at late zygotene, but also showed considerable gene-to-gene variability in silencing dynamics (Fig. 5A). Four sets of these genes formed distinct waves of transcription. The largest wave contains genes expressed in spermatogonia (cluster 1). 
This, in turn, is consistent with the observations that the $\mathrm{X}$ chromosome is enriched in premeiotic genes (Khil et al., 2004; Koslowski et al., 2006). Yet, interestingly, many other genes were transiently induced prior to $\mathrm{MSCl}$. The combination of these patterns (gradual silencing \& pre-MSCI transcriptional burst) also clarifies why the total expression from both the sex chromosomes ramps up before $\mathrm{MSCl}$ (Fig. 5B). We also confirmed our observation using a recently published dataset (Ernst et al., 2019).

We also noticed that the sex chromosomal expression clearly anti-correlated with the expression of H2afx (Fig. 5B, stages marked by a color bar on top). This observation contrasts with the ubiquitous deposition of $H 2 a f x$ along the genome, and with the idea that is mostly regulated post-transcriptionally (Turner et al., 2004; Bellani et al., 2005; Turinetto and Giachino, 2015). H2afx can be phosphorylated by Atm, Atr and Pkrdc, and we confirmed that only Atr is expressed during MSCI (Bellani et al., 2005), however it shows decreasing mRNA levels (Fig. 5C). The lack Atr induction around $\mathrm{MSCl}$ alongside the observation that $\mathrm{H} 2 \mathrm{afx}$ is induced precisely at this time suggests that the timing of $\mathrm{MSCl}$ is partly controlled by H2afx transcription. Possibly, then the main role of the Atr could be to activate the newly deposited histones.

It has been proposed that $\mathrm{MSCl}$ is simply a selective lack of reactivation of the sex chromosomes during pachytene (Page et al. 2012). Here, we show that $\mathrm{MSCl}$ is an separate silencing event (Fig. 5B). First of all, sex chromosomal transcription sharply falls before pachytene activation. Second, many sex chromosomal genes were specifically upregulated before silenced (Fig. 5A). Finally, transcript levels after (pre)leptotene silencing are stable until pachytene, except from the sex chromosomes (Fig. 2D).

Some $\mathrm{X}$ chromosomal genes have autosomal paralogs, which switch expression to the autosomal copy upon MSCl. We indeed see the timing and extent of autosomal compensation in our data. As examples, we detected that when the $\mathrm{X}$ chromosomal gene Rp/10 is silenced by MSCI, its paralog, Rp/10L on chr12 is induced (Jiang et al., 2017). The paralog pairs Pgk1 (chrX) - Pgk2 (chr17), and the Cetn2 (chrX) - Cetn1 (chr18) (Hart et al., 1999) show the same trend (Data S6).

It is unknown whether MSCI follows any pattern and if genes are silenced in any particular order. The best-studied example of chromosome-wide silencing is the $\mathrm{X}$ chromosome inactivation $(\mathrm{XCl})$ in female mice. Although $\mathrm{XCl}$ evolved to achieve dosage compensation, both involve the silencing of the whole $\mathrm{X}$ chromosome. Silencing in $\mathrm{XCl}$ is primarily 
governed by chromosome structure and proximity to the X-inactivation center (Nora et al., 2012; Engreitz et al., 2013).

We therefore tested the possibility of a silencing center, or a silencing bias towards either chromosomal end. In the telocentric chromosomes of mice, centromeres occupy one end of each chromosome. For the $X$ and $Y$ chromosomes, the other end forms the pseudoautosomal region (PAR). While the centromere holds the sister chromatids together, the PAR holds the $X \& Y$ chromosomes together in meiosis. Thus, both ends might bias chromatin silencing.

We then analyzed the silencing dynamics along the $\mathrm{X}$ chromosome, on a linear basis. While we found ample variability in silencing dynamics, we detected no spatial effect towards either end of the $\mathrm{X}$ chromosome, nor towards the Xist locus (Data S4). Thus, we concluded that unlike $\mathrm{X}$-inactivation, $\mathrm{MSCl}$ is unlikely to be governed by proximity to an inactivation center.

Genes in the pseudoautosomal regions (PAR) are X/Y homologous, and not silenced, so they could be used as an internal negative control for $\mathrm{MSCl}$. In mice however, PAR only contains 3 active genes (Kasahara et al., 2010). While Sts, and Asmt (Hiomt) transcripts were not detected, Mid1 (Fxy) indeed showed low, but constant raw transcript counts throughout prophase-I (Fig. S6E).

We further examined the expression of the latest silenced genes in the second half of $\mathrm{MSCl}$ (cluster 4). We found a small set of genes with remarkably high expression (Fig. 5D). These genes -except Prdx4- were specifically induced in zygotene and showed high expression, when the expression of other sex chromosomal genes was already decreasing (Fig. 5E). Scm/2 is a testis-specific polycomb protein, which regulates the ubiquitination state of H2afx. It showed one of the strongest and latest expression peak, underlining its important role in controlling MSCI (Luo et al., 2015) and genes escaping silencing (Adams et al. 2018).

Based on the functional cues among the late-silenced genes, we performed a comprehensive analysis of gene function during silencing. We classified sex chromosomal genes into fast- and slow-silenced categories based on their characteristic silencing time (Online Methods). For each group, we calculated GO-term enrichment as compared to all sex chromosomal genes (Online Methods). We found that while early-silenced genes were typically localized to the nucleus ( $43 \%$ of the genes, FDR $<4 \mathrm{e}^{-3}$ ). Inversely, latesilenced genes were preferentially localized to the cytoplasm $\left(56 \%\right.$, FDR $\left.<2 e^{-6}\right)$ and were 
enriched in genes encoding mitochondrial proteins $\left(19 \%\right.$, FDR $\left.<2 e^{-5}\right)$. This further supported the notion that silencing is a coordinated process starting with regulatory genes and followed by cytoplasmic and metabolic genes (catalytic activity: $34 \%$ of late genes, FDR $\left.<8 \mathrm{e}^{-3}\right)($ Table S4).

\section{Slitrk2 and other genes escape MSCl}

We also noticed that the Ndufa1 (Fig. 5D), is not only the last gene to be downregulated, but it also maintains a steady absolute transcript level throughout pachytene, possibly by escaping inactivation. Whether genes escape $\mathrm{MSCl}$ is debated in recent literature (Chen et al., 2018; Jung et al., 2018). Transcripts detected in pachytene can either be due to mRNA preservation over several days, or due to an active transcription from $\mathrm{MSCl}$. Therefore, it is important to characterize if such genes show increased expression after $\mathrm{MSCl}$, and to validate their expression pattern independently.

To examine if other sex chromosomal genes are also detected after $\mathrm{MSCl}$, we calculated the average raw transcript counts in cluster 5 (as normalized counts may be misleading, see Fig. 2D and Online Methods). We identified 27 genes on chromosome $X$ with above and an average of one transcript per cell (Fig. 5G). This correspond to approximately 10100 transcripts per cell, given the estimated sensitivity of single-cell methods (Grün, Kester and van Oudenaarden, 2014). Importantly, we detected more transcripts for these 27 genes after $\mathrm{MSCl}$, suggesting that their expression is not simply due to mRNA preservation.

Slitrk2 showed the most striking expression pattern, as it was specifically upregulated after MSCl (Fig. 5G). Slitrk2 is the only gene expressed from the hybrid sterility locus (Hstx1/2). It has been described to escape silencing in domesticus $X$ musculus hybrids (Bhattacharyya et al., 2014). It is not expressed in Sertoli cells (Zimmermann et al., 2015), and we validated its expression in vivo by smFISH (Fig. 5J-K).

Mapping these 27 escapee genes on the $X$ chromosome showed a clustered pattern around the Zfx, Irak1, and Xist loci (Fig. 5H). Note, that Xist itself is not expressed in males. ATAC-seq has previously identified the Xist locus as one of the most open chromatin regions during $\mathrm{XCl}$ in females (Giorgetti et al., 2016), which may contribute to the lack of silencing in genes located in proximity of Xist. In addition, we noticed that many of the escaping genes from the Xist locus are long, and often non-coding genes. Indeed, 
352 the transcript length of the 27 escapee genes are significantly longer than other $X$ 353 chromosomal genes (one-sided MWW test, $\mathrm{p}<0.006$ ).

354 When compared to known escapees in post-meiotic sex chromosome inactivation 355 (Namekawa et al., 2006) and MSCI, only Mageb.ps-1 is shared. Similarly, when compared $38 \mathrm{XCl}$ escapees (Berletch et al., 2015) with $\mathrm{MSCl}$, only Car5b was common (Fig. 5I). These observations suggest that a mechanism of silencing and escaping distinct from previously studied cases (e.g. $\mathrm{XCl}$ ) occurs during the meiotic phase.

Finally, we wanted to know if escapee genes show specific expression dynamics during prophase. Therefore, we clustered gene expression profiles along the trajectory. We found that most genes follow a pattern similar to Ndufa1, while the expression pattern of Slitrk2 is unique (Fig. S5A). Yet, both patterns appeared as residual rather than functional. This is because relative transcript counts -within the expanding pachytene transcriptomeremained in all cases low. Escape from silencing then might simply be the consequence of open chromatin amidst global transcription. An exception to this might be Slitrk2.

Taken together, we found that a third of sex chromosomal genes are specifically induced before silencing by $\mathrm{MSCl}$. Although $\mathrm{MSCl}$ is a short event in meiosis, we found remarkable differences in the silencing time of genes. While broad chromosome structure does not appear to affect silencing time, we found functional cues. early-silenced genes are mostly nuclear, but late-silenced genes are often cytoplasmic or mitochondrial. This leads to the interesting possibility of a "regulation first, processing second" silencing pattern. Yet, to understand the role of $\mathrm{MSCl}$, one must consider a combination of ancestral and newly acquired functions (Turner et al., 2004). MSCI might have evolved for one reason, e.g. global silencing of unsynapsed chromatin, and once in place, it could evolve to new regulatory functions. anatomy.

The transient silencing during leptotene and zygotene ends before pachytene (Monesi, 1965). This leads up to 9-times more cellular mRNA than in earlier stages (Fig. 2D, inset, at 900 on the trajectory). In accord with a global transcriptional activation, cells in pachytene reach the most diverse transcriptome in our dataset, as quantified by entropy 
To characterize this massive transcriptional wave, we first identified genes upregulated in pachytene by differential gene expression analysis (Fig. 6A). We selected all genes that showed a 3-fold upregulation compared to all other cells (Online Methods). We classified these into 3 categories by k-means clustering of z-score normalized rolling average expression profiles (Fig. 6D). Clusters showed reproducibility $>98 \%$ over 10000 bootstrapping rounds, and gene classes displayed sharp, distinct activation profiles (Fig. $6 B \& E)$.

Many immediate genes had functions linked to the pachytene transcriptional activation itself. Other immediate genes encoded proteins for energy production (e.g. The mitochondrial cytochrome c oxidase genes) (Fig. 6C). Piwi-pathway related genes formed the next wave in the 'intermediate class of genes (Piwil1, Tdrd1, Pld6 and Gtsf1), protecting against transposons (Aravin, Hannon and Brennecke, 2007).

Yet, most genes activated in pachytene have post-meiotic roles (Da Cruz, 2016). Many of the enriched functions are first relevant in elongating spermatid (e.g. flagella formation) or in mature spermatozoa (e.g. binding to zona pellucida). These genes are thus surprisingly transcribed more than a week before their utilization. The Adam gene family (Primakoff and Myles, 2000) is a typical example of the pachytene-activated, fertilization-related genes. One of these gradual genes is Adam32 (Fig. 6F). We validated its expression by smFISH (Fig. 6I), which also matched the expression pattern found in neonatal testis (Choi et al., 2003).

We also found distinct functional signature of immediate and gradual genes, while the intermediate class of genes showed a blend of functional enrichments (Fig. 6G). Interestingly, the genes were activated in parallel order to the order they will be used in the mature spermatozoa. While immediate genes play a role in flagella formation and cell movement, late genes are involved in fertilization (Table. S6). Accordingly, enriched GOterms in the immediate and gradual classes mapped to different anatomical regions of the spermatozoa (Fig. 6H).

Surprisingly, while these genes are transcribed in a precise order, they are only translated and used more than a week later. This temporal discrepancy highlights the important role of mRNA storage in spermatogenesis. In accord, we noted that pachytene activation is exactly the time when Dazl, an RNA binding protein, is downregulated (Fig. 1D). Altogether, we observed a sequential pattern of gene activation in pachytene cells that 
416 reflect the spatial anatomy of the mature sperm. This temporal pattern might show a spatial pattern of maturation of the germ cell, from the tail to the head.

\section{Discussion}

419 Currently, meiotic entry in mammals is not well understood, as highlighted by our inability 420 to induce meiosis purely in vitro, without the co-culture of explanted tissues. To gain a 421 transcriptome-wide and time-resolved insight, multiple recent studies applied single-cell mRNA sequencing combined with pseudo-temporal reconstruction (Chen et al., 2018; Green et al., 2018; Hermann et al., 2018; Jung et al., 2018; Lukassen et al., 2018; Ernst et al., 2019). These studies provided excellent resources and greatly contributed to understand the whole span of spermatogenesis. Yet, additional questions require deeper, fine-tuned, computational analysis.

Our study focused on early meiosis up until pachytene. We found that in contrast with the multitude of morphological stages, there are only three major transcriptomic events. In the first major transition spermatogonia committed to meiosis. This is accompanied by a global shrinkage of the transcriptome and a clear functional switch. Cells shift from a mitotic and catabolic to a meiotic and recombination-related transcriptome. This functional switch however appears to be regulated by a one-sided silencing of a transcription factors hub around $n-M y c$, while no comparable network is upregulated.

434 The second major transition is meiotic sex chromosome inactivation. It is thought that the phosphorylation of the silencing histone variant H2afx controls the timing of inactivation. We however found that $\mathrm{H}$ 2afx gene is induced exactly during $\mathrm{MSCl}$ and it is anti-correlated to sex chromosomal expression. These together suggest that the timing of $\mathrm{MSCl}$ is controlled by the surplus of H2afx expression. At the same time Atr expression is flat, which may hint towards no temporal control on the post transcriptional level.

We then characterized chromosome-wide activation and silencing patterns before, and during $\mathrm{MSCl}$. We found that sex chromosomal genes are silenced at distinct times, challenging established models for $\mathrm{MSCl}$. For example, if repression would only appear 443 to protect DNA, we would not expect that genes silence in a coordinated, sequential manner.

445 In general, silencing follows two major patterns: gradual silencing and transient activation 446 followed by silencing. We found that $34 \%$ of sex chromosomal genes were specifically 
induced before $\mathrm{MSCl}$. We speculate that some of these gene products may serve as buffer or pool until autosomal genes take over their functions in pachytene. Additionally, many of these transiently activated genes were involved in regulating gene expression. Interestingly, the chromatin regulator Scm/2 (Hasegawa et al., 2015; Luo et al., 2015; Adams et al., 2018) is one of the genes strongest induced before, and latest silenced upon $\mathrm{MSCl}$, highlighting that gene function and silencing time are concerted. In addition, genes activated just before $\mathrm{MSCl}$ might act as a trigger for the third major transition, the pachytene activation.

Taken together, sex chromosomes are silenced in a functional, rather than structural order, raising the possibility that $\mathrm{MSCl}$ is involved in gene regulation. This, in turn, could also explain why $\mathrm{MSCl}$ is essential for meiotic program. Nevertheless, we think there is no single purpose behind $\mathrm{MSCl}$ : gene regulation could have evolved atop of its ancestral functions. To deeper understand $\mathrm{MSCl}$, one could complement chromatin profiling with single-cell mRNA-seq, as for PMSCI (Ernst et al., 2019), or perform single-cell chromatin accessibility profiling.

Finally, the third major transition in prophase-I is pachytene activation. This global transcriptional activation boosts cellular mRNA content up to 9-fold. We found that immediately and gradually activated genes are functionally distinct. While immediate genes were related to energy metabolism and spermatozoal movement, gradual genes were related to fertilization. Accordingly, the corresponding gene products mapped exclusively to the posterior, or anterior parts of the mature sperm.

In sum, we elucidated key events in of early meiosis, and provided a transcriptome-wide, precisely timed, gene expression atlas for online exploration. Our approach hopefully will be followed by functional studies showing the importance of timing in meiosis.

\section{Acknowledgements}

We would like to thank Mauro help for experimental help, Willy Baarends and Joost Gribnau for valuable comments on the manuscript, and help with histological analysis, Stefan van der Elst and Reinier van der Linden for help with FACS sorting, Nicolas Battich for advices on microscopy analysis, Josi Peterson-Maduro for logistic help and Fanny Sage for the mouse drawing. 


\section{References}

Adams, S. R. et al. (2018) 'RNF8 and SCML2 cooperate to regulate ubiquitination and H3K27 acetylation for escape gene activation on the sex chromosomes', PLoS Genetics, 14(2). doi: 10.1371/journal.pgen.1007233.

Anders, S. and Huber, W. (2010) 'Differential expression analysis for sequence count data', Genome biology, 11(R106), pp. 1-12. doi: 10.1186/gb-2010-11-10-r106.

Anderson, E. L. et al. (2008) 'Stra8 and its inducer, retinoic acid, regulate meiotic initiation in both spermatogenesis and oogenesis in mice', Proceedings of the National Academy of Sciences, 105(39), pp. 14976-14980. doi: 10.1073/pnas.0807297105.

Aravin, A. a, Hannon, G. J. and Brennecke, J. (2007) 'The Piwi-piRNA pathway provides an adaptive defense in the transposon arms race.', Science (New York, N.Y.), 318(5851), pp. 7614. doi: $10.1126 /$ science.1146484.

Baarends, W. M. et al. (2005) 'Silencing of unpaired chromatin and histone H2A ubiquitination in mammalian meiosis.', Molecular and cellular biology, 25(3), pp. 1041-53. doi: 10.1128/MCB.25.3.1041-1053.2005.

Bellani, M. A. et al. (2005) 'SPO11 is required for sex-body formation, and Spo11 heterozygosity rescues the prophase arrest of Atm-/- spermatocytes.', Journal of cell science, 118(Pt 15), pp. 3233-45. doi: 10.1242/jcs.02466.

Bengtsson, M. et al. (2005) 'Gene expression profiling in single cells from the pancreatic islets of Langerhans reveals lognormal distribution of mRNA levels', Genome research, pp. 1388-1392. doi: $10.1101 / \mathrm{gr} .3820805 .4$.

Berletch, J. B. et al. (2015) 'Escape from X inactivation varies in mouse tissues.', PLoS genetics. Edited by M. S. Bartolomei, 11(3), p. e1005079. doi: 10.1371/journal.pgen.1005079.

Bhattacharyya, T. et al. (2014) 'X Chromosome Control of Meiotic Chromosome Synapsis in Mouse Inter-Subspecific Hybrids', PLoS Genetics. Edited by B. A. Payseur, 10(2), p. e1004088. doi: 10.1371/journal.pgen.1004088.

Binns, D. et al. (2009) 'QuickGO: a web-based tool for Gene Ontology searching', Bioinformatics. Oxford University Press, 25(22), pp. 3045-3046. doi: 10.1093/bioinformatics/btp536.

Braydich-Stolle, L. et al. (2007) 'Role of Src family kinases and N-Myc in spermatogonial stem cell proliferation', Developmental Biology, 304(1), pp. 34-45. doi: 10.1016/j.ydbio.2006.12.013.

Van Den Brink, S. C. et al. (2017) 'Single-cell sequencing reveals dissociation-induced gene expression in tissue subpopulations', Nature Methods, 14(10). doi: 10.1038/nmeth.4437.

Cannoodt, R., Saelens, W. and Saeys, Y. (2016) 'Computational methods for trajectory inference from single-cell transcriptomics', European Journal of Immunology, 46(11), pp. 24962506. doi: 10.1002/eji.201646347.

Carbon, S. et al. (2009) 'AmiGO: online access to ontology and annotation data', Bioinformatics. Oxford University Press, 25(2), pp. 288-289. doi: 10.1093/bioinformatics/btn615.

Chakraborty, P. et al. (2014) 'LIN28A Marks the Spermatogonial Progenitor Population and Regulates Its Cyclic Expansion', STEM CELLS, 32(4), pp. 860-873. doi: 10.1002/stem.1584.

Chen, E. Y. et al. (2013) 'Enrichr: interactive and collaborative HTML5 gene list enrichment analysis tool', BMC Bioinformatics, 14(1), p. 128. doi: 10.1186/1471-2105-14-128.

Chen, H. H. et al. (2014) 'DAZL limits pluripotency, differentiation, and apoptosis in developing primordial germ cells', Stem Cell Reports, 3(5), pp. 892-904. doi: 10.1016/j.stemcr.2014.09.003. 
Chen, Y. et al. (2018) 'Single-cell RNA-seq uncovers dynamic processes and critical regulators in mouse spermatogenesis', Cell Research, 28(9), pp. 879-896. doi: 10.1038/s41422-018-0074y.

Choi, I. et al. (2003) 'Identification and characterization of ADAM32 with testis-predominant gene expression', Gene, 304(1-2), pp. 151-162. doi: 10.1016/S0378-1119(02)01202-7.

da Cruz, I. et al. (2016) 'Transcriptome analysis of highly purified mouse spermatogenic cell populations: gene expression signatures switch from meiotic-to post-meiotic-related processes at pachytene stage.', BMC genomics, 17(1), p. 294. doi: 10.1186/s12864-016-2618-1.

Engreitz, J. M. et al. (2013) 'The Xist IncRNA Exploits Three-Dimensional Genome Architecture to Spread Across the X Chromosome', Science, 341(6147), pp. 1237973-1237973. doi: 10.1126/science.1237973.

Ernst, C. et al. (2019) 'Staged developmental mapping and X chromosome transcriptional dynamics during mouse spermatogenesis', Nature Communications, 10, p. 1251. doi: 10.1038/s41467-019-09182-1.

Fallahi, M. et al. (2010) 'A Global Expression Switch Marks Pachytene Initiation during Mouse Male Meiosis', Genes, 1(3), pp. 469-483. doi: 10.3390/genes1030469.

Feng, X. H., Lin, X. and Derynck, R. (2000) 'Smad2, Smad3 and Smad4 cooperate with Sp1 to induce p15(Ink4B) transcription in response to TGF-beta.', The EMBO journal, 19(19), pp. 517893. doi: 10.1093/emboj/19.19.5178.

Fernandez, P. C. (2003) 'Genomic targets of the human c-Myc protein', Genes \& Development, 17(9), pp. 1115-1129. doi: 10.1101/gad.1067003.

Giorgetti, L. et al. (2016) 'Structural organization of the inactive X chromosome in the mouse.', Nature, in press(7613), pp. 1-5. doi: 10.1038/nature18589.

Goldberg, E. et al. (2010) 'LDHC: The Ultimate Testis-Specific Gene', Journal of Andrology, 31(1), pp. 86-94. doi: 10.2164/jandrol.109.008367.

Goymer, P. (2005) 'Which mean do you mean?', Nature genetics, 6(December), pp. 2005-2005. doi: 10.1080/0020739860170303.

Green, C. D. et al. (2018) 'A Comprehensive Roadmap of Murine Spermatogenesis Defined by Single-Cell RNA-Seq', Developmental Cell, 46(5), p. 651-667.e10. doi:

10.1016/j.devcel.2018.07.025.

Griswold, M. D. (2016) 'Spermatogenesis: The Commitment to Meiosis', Physiological Reviews, 96(1), pp. 1-17. doi: 10.1152/physrev.00013.2015.

Grün, D. et al. (2015) 'Single-cell messenger RNA sequencing reveals rare intestinal cell types', Nature. Nature Research, 525(7568), pp. 251-255. doi: 10.1038/nature14966.

Grün, D. et al. (2016) 'De Novo Prediction of Stem Cell Identity using Single-Cell Transcriptome Data', Cell Stem Cell, 19(2), pp. 266-277. doi: 10.1016/j.stem.2016.05.010.

Grün, D., Kester, L. and van Oudenaarden, A. (2014) 'Validation of noise models for single-cell transcriptomics.', Nature methods, 11(6), pp. 637-40. doi: 10.1038/nmeth.2930.

Grun, D. and van Oudenaarden, A. (2015) 'Design and Analysis of Single-Cell Sequencing Experiments.', Cell, 163(4), pp. 799-810. doi: 10.1016/j.cell.2015.10.039.

Hart, P. E. et al. (1999) 'Testis-Specific Murine Centrin, Cetn1: Genomic Characterization and Evidence for Retroposition of a Gene Encoding a Centrosome Protein', Genomics, 60(2), pp. 111-120. doi: 10.1006/geno.1999.5880. 
Regulation of Histone H2A Ubiquitination', Developmental Cell, 32(5), pp. 574-588. doi: 10.1016/j.devcel.2015.01.014.

Hashimshony, T. et al. (2012) 'CEL-Seq: Single-Cell RNA-Seq by Multiplexed Linear Amplification', Cell Reports. The Authors, 2(3), pp. 666-673. doi: 10.1016/j.celrep.2012.08.003.

Hashimshony, T. et al. (2016) 'CEL-Seq2: sensitive highly-multiplexed single-cell RNA-Seq', Genome Biology. BioMed Central, 17(1), p. 77. doi: 10.1186/s13059-016-0938-8.

Hastie, T. and Stuetzle, W. (1989) 'Principal Curves', Journal of the American Statistical Association. Taylor \& Francis, 84(406), pp. 502-516. doi: 10.1080/01621459.1989.10478797.

van der Heijden, G. W. et al. (2007) 'Chromosome-wide nucleosome replacement and H3.3 incorporation during mammalian meiotic sex chromosome inactivation', Nature Genetics, 39(2), pp. 251-258. doi: 10.1038/ng1949.

Henderson, S. A. (1963) 'Differential Ribonucleic Acid Synthesis Of X And Autosomes During Meiosis.', Nature, 200, p. 1235. doi: 14089938.

Hermann, B. P. et al. (2018) 'The Mammalian Spermatogenesis Single-Cell Transcriptome, from Spermatogonial Stem Cells to Spermatids', Cell Reports, 25(6), p. 1650-1667.e8. doi: 10.1016/j.celrep.2018.10.026.

Jan, S. Z. et al. (2017) 'Unraveling transcriptome dynamics in human spermatogenesis', Development, p. dev.152413. doi: 10.1242/dev.152413.

Ji, Z. and Ji, H. (2016) 'TSCAN: Pseudo-time reconstruction and evaluation in single-cell RNAseq analysis', Nucleic Acids Research, 44(13), p. e117. doi: 10.1093/nar/gkw430.

Jiang, L. et al. (2017) 'RPL10L Is Required for Male Meiotic Division by Compensating for RPL10 during Meiotic Sex Chromosome Inactivation in Mice', Current Biology, 27(10), p. 14981505.e6. doi: 10.1016/j.cub.2017.04.017.

Jung, M. et al. (2018) 'Unified single-cell analysis of testis gene regulation and pathology in 5 mouse strains', bioRxiv, p. 393769. doi: 10.1101/393769.

Kasahara, T. et al. (2010) 'Genetic variation of melatonin productivity in laboratory mice under domestication', Proceedings of the National Academy of Sciences, 107(14), pp. 6412-6417. doi: 10.1073/pnas.0914399107.

Khalil, A. M., Boyar, F. Z. and Driscoll, D. J. (2004) 'Dynamic histone modifications mark sex chromosome inactivation and reactivation during mammalian spermatogenesis', Proc Natl Acad Sci U S A, 101(47), pp. 16583-16587. doi: 10.1073/pnas.0406325101.

Khil, P. P. et al. (2004) 'The mouse X chromosome is enriched for sex-biased genes not subject to selection by meiotic sex chromosome inactivation', Nature Genetics, 36(6), pp. 642-646. doi: $10.1038 / n g 1368$.

Kim, B., Cooke, H. J. and Rhee, K. (2012) 'DAZL is essential for stress granule formation implicated in germ cell survival upon heat stress', Development, 139, pp. 568-578. doi: 10.1242/dev.075846.

Koslowski, M. et al. (2006) 'The human X chromosome is enriched for germline genes expressed in premeiotic germ cells of both sexes', Human Molecular Genetics, 15(15), pp. 2392-2399. doi: 10.1093/hmg/ddl163.

Kuleshov, M. V. et al. (2016) 'Enrichr: a comprehensive gene set enrichment analysis web server 2016 update', Nucleic acids research, 44(W1), pp. W90-W97. doi: 10.1093/nar/gkw377.

Li, H. (2013) 'Aligning sequence reads, clone sequences and assembly contigs with BWA-MEM'. Lukassen, S. et al. (2018) 'Characterization of germ cell differentiation in the male mouse 
through single-cell RNA sequencing', Scientific Reports, 8(1), p. 6521. doi: 10.1038/s41598-01824725-0.

Luo, J., Megee, S. and Dobrinski, I. (2009) 'Asymmetric Distribution of UCH-L1 in Spermatogonia Is Associated With Maintenance and Differentiation of Spermatogonial Stem Cells', Journal of Cellular Physiology, 220(2), pp. 460-468. doi: 10.1002/jcp.21789.

Luo, M. et al. (2013) 'MEIOB exhibits single-stranded DNA-binding and exonuclease activities and is essential for meiotic recombination.', Nature communications, 4(1), p. 2788. doi: $10.1038 /$ ncomms 3788 .

Luo, M. et al. (2015) 'Polycomb protein SCML2 associates with USP7 and counteracts histone $\mathrm{H} 2 \mathrm{~A}$ ubiquitination in the XY chromatin during male meiosis.', PLoS genetics. Edited by J. C. Schimenti. Public Library of Science, 11(1), p. e1004954. doi: 10.1371/journal.pgen.1004954.

Mahadevaiah, S. K. et al. (2001) 'Recombinational DNA double-strand breaks in mice precede synapsis.', Nature genetics. Nature Publishing Group, 27(3), pp. 271-6. doi: 10.1038/85830.

Mao, Q. et al. (2016) 'Principal Graph and Structure Learning Based on Reversed Graph Embedding', IEEE Transactions on Pattern Analysis and Machine Intelligence, XX(X), pp. 1-1. doi: 10.1109/TPAMI.2016.2635657.

Margolin, G. et al. (2014) 'Integrated transcriptome analysis of mouse spermatogenesis', BMC Genomics, 15(1), p. 39. doi: 10.1186/1471-2164-15-39.

Matson, C. K. et al. (2010) 'The Mammalian Doublesex Homolog DMRT1 Is a Transcriptional Gatekeeper that Controls the Mitosis versus Meiosis Decision in Male Germ Cells', Developmental Cell, 19(4), pp. 612-624. doi: 10.1016/j.devcel.2010.09.010.

Matys, V. (2006) 'TRANSFAC(R) and its module TRANSCompel(R): transcriptional gene regulation in eukaryotes', Nucleic Acids Research, 34(90001), pp. D108-D110. doi: 10.1093/nar/gkj143.

McKee, B. D. and Handel, M. A. (1993) 'Sex chromosomes, recombination, and chromatin conformation', Chromosoma, 102(2), pp. 71-80. doi: 10.1007/BF00356023.

Meistrich, M. L. and Hess, R. A. (2013) 'Assessment of Spermatogenesis Through Staging of Seminiferous Tubules', in Carrell, D. T. and Aston, K. I. (eds) Spermatogenesis: Methods and Protocols. Totowa, NJ: Humana Press, pp. 299-307. doi: 10.1007/978-1-62703-038-0_27.

Mering, C. v. (2003) 'STRING: a database of predicted functional associations between proteins', Nucleic Acids Research. Oxford University Press, 31(1), pp. 258-261. doi: 10.1093/nar/gkg034.

Monesi, V. (1965) 'Differential rate of ribonucleic acid synthesis in the autosomes and sex chromosomes during male meiosis in the mouse', Chromosoma, 17(1), pp. 11-21. doi: 10.1007/BF00285153.

Muraro, M. J. et al. (2016) 'A Single-Cell Transcriptome Atlas of the Human Pancreas', Cell Systems, 3(4), p. 385-394.e3. doi: 10.1016/j.cels.2016.09.002.

Nakata, H. et al. (2015) 'Quantitative analysis of the cellular composition in seminiferous tubules in normal and genetically modified infertile mice.', The journal of histochemistry and cytochemistry: official journal of the Histochemistry Society, 63(2), pp. 99-113. doi: 10.1369/0022155414562045.

Namekawa, S. H. et al. (2006) 'Post-meiotic Sex Chromatin in the Male Germline of Mice', Current Biology, 16(7), pp. 660-667. doi: 10.1016/j.cub.2006.01.066.

Nguyen, T. B. et al. (2002) 'Characterization and Expression of Mammalian Cyclin B3, a Prepachytene Meiotic Cyclin', Journal of Biological Chemistry, 277(44), pp. 41960-41969. doi: 
Nora, E. P. et al. (2012) 'Spatial partitioning of the regulatory landscape of the X-inactivation centre', Nature, 485(7398), pp. 381-385. doi: 10.1038/nature11049.

O'Shaughnessy, P. J., Morris, I. D. and Baker, P. J. (2008) 'Leydig cell re-generation and expression of cell signaling molecules in the germ cell-free testis', Reproduction, 135(6), pp. 851-858. doi: 10.1530/REP-07-0529.

Page, J. et al. (2012) 'Inactivation or non-reactivation: what accounts better for the silence of sex chromosomes during mammalian male meiosis?', Chromosoma, 121(3), pp. 307-326. doi: 10.1007/s00412-012-0364-y.

Petropoulos, S. et al. (2016) 'Single-Cell RNA-Seq Reveals Lineage and X Chromosome Dynamics in Human Preimplantation Embryos.', Cell, 165(4), pp. 1012-26. doi: 10.1016/j.cell.2016.03.023.

Portales-Casamar, E. et al. (2010) 'JASPAR 2010: the greatly expanded open-access database of transcription factor binding profiles.', Nucleic acids research. Oxford University Press, 38(Database issue), pp. D105-10. doi: 10.1093/nar/gkp950.

Primakoff, P. and Myles, D. G. (2000) 'The ADAM gene family: Surface proteins with adhesion and protease activity', Trends in Genetics, 16(2), pp. 83-87. doi: 10.1016/S01689525(99)01926-5.

Qiu, X. et al. (2017) 'Reversed graph embedding resolves complex single-cell trajectories.', Nature methods, 14(10), pp. 979-982. doi: 10.1038/nmeth.4402.

Raivo, K. (2013) pheatmap: Pretty Heatmaps.

Royo, H. et al. (2015) 'Silencing of X-Linked MicroRNAs by Meiotic Sex Chromosome Inactivation', PLoS Genetics, 11(10), pp. 1-15. doi: 10.1371/journal.pgen.1005461.

Russell, L. D. et al. (1993) Histological and Histopathological Evaluation of the Testis. doi: 10.1111/j.1365-2605.1993.tb01156.x.

Shin, Y.-H. et al. (2010) 'Hormad1 mutation disrupts synaptonemal complex formation, recombination, and chromosome segregation in mammalian meiosis.', PLoS genetics. Edited by R. S. Hawley, 6(11), p. e1001190. doi: 10.1371/journal.pgen.1001190.

Shiu, P. K. T. et al. (2001) 'Meiotic Silencing by Unpaired DNA', Cell, 107(7), pp. 905-916. doi: 10.1016/S0092-8674(01)00609-2.

Sun, F. et al. (2015) 'Nuclear localization of PRDM9 and its role in meiotic chromatin modifications and homologous synapsis.', Chromosoma, 124(3), pp. 397-415. doi: 10.1007/s00412-015-0511-3.

Supek, F. et al. (2011) 'Revigo summarizes and visualizes long lists of gene ontology terms', PLOS ONE. Edited by C. Gibas, 6(7), p. e21800. doi: 10.1371/journal.pone.0021800.

Szklarczyk, D. et al. (2017) 'The STRING database in 2017: quality-controlled protein-protein association networks, made broadly accessible', Nucleic Acids Research. Oxford University Press, 45(D1), pp. D362-D368. doi: 10.1093/nar/gkw937.

Tibshirani, R., Walther, G. and Hastie, T. (2001) 'Estimating the number of clusters in a data set via the gap statistic', Journal of the Royal Statistical Society: Series B (Statistical Methodology), 63(2), pp. 411-423. doi: 10.1111/1467-9868.00293.

Turinetto, V. and Giachino, C. (2015) 'Multiple facets of histone variant H2AX: a DNA doublestrand-break marker with several biological functions.', Nucleic acids research, 43(5), pp. 248998. doi: 10.1093/nar/gkv061. 
Turner, J. M. A. et al. (2004) 'BRCA1, Histone H2AX Phosphorylation, and Male Meiotic Sex Chromosome Inactivation', Current Biology, 14(23), pp. 2135-2142. doi: 10.1016/j.cub.2004.11.032.

Turner, J. M. A. (2015) 'Meiotic Silencing in Mammals.', Annual review of genetics, 49(1), pp. 395-412. doi: 10.1146/annurev-genet-112414-055145.

Vandesompele, J. et al. (2002) 'Accurate normalization of real-time quantitative RT-PCR data by geometric averaging of multiple internal control genes', Genome Biology, 3(7), p. research0034.1. doi: 10.1186/gb-2002-3-7-research0034.

Ventela, S. et al. (2000) 'Expression of green fluorescent protein under beta-actin promoter in living spermatogenic cells of the mouse: stage-specific regulation by FSH', International Journal of Andrology, 23(4), pp. 236-242. doi: 10.1046/j.1365-2605.2000.00237.x.

Vertesy, A. (2017) 'MarkdownReports: An R function library to create scientific figures and markdown reports easily. Update v2.9.5'. doi: 10.5281/zenodo.1014353.

Wang, J. et al. (2017) 'NRF1 coordinates with DNA methylation to regulate spermatogenesis', The FASEB Journal, 31(11), pp. 4959-4970. doi: 10.1096/fj.201700093R.

Xue, H. et al. (1999) 'HemT-3, an alternative transcript of mouse gene HemT specific to male germ cells.', Gene, 240(1), pp. 193-9. doi: 10.1016/S0378-1119(99)00401-1.

Yoshinaga, K. et al. (1991) 'Role of c-kit in mouse spermatogenesis: identification of spermatogonia as a specific site of c-kit expression and function.', Development (Cambridge, England), 113(2), pp. 689-99. Sertoli Cells During the Progression of Spermatogenesis', Molecular Endocrinology, 29(4), pp. 627-642. doi: 10.1210/me.2014-1356. 
Fig.1: Single cell mRNA sequencing identifies five subtypes of Dazl-GFP+ germ cells, which correspond to subsequent stages of prophase-I.

a.) Experimental and analytic workflow.

b.) Transcriptome-wide k-medoids clustering identifies 5 clusters among 1274 high quality Dazl-GFP+ cells. Spearman correlation coefficients show homogenous and distinct clusters (cl. $1 \& 5$ ), while the three other stages are more heterogeneous.

c.) Cell-to-cell differences visualized on t-SNE map. Stages along the curve from left (1) to right (5) represent consecutive stages of prophase-I. Colors and numbers indicate clusters (as determined in panel B) and represent spermatogonial (1), (pre)leptotene I (2), zygotene (3), and pachytene $(4,5)$ stages. Each dot represents a single cell.

d.) Expression of the selected marker genes. Negative and positive controls: No sorted cells express the Sertoli cell specific retinol binding protein 4 receptor (Stra6) or the Leydig cell specific anti-Mullerian hormone $(A m h)$, but all cells express the sorting marker Dazl-GFP mRNA, validating sorting approach. Synaptonemal Complex Protein 3 (Sycp3) is a core component of the synaptonemal complex connecting homologous chromosomes in meiotic prophase. $\mathbf{C l}$. 1 cells express spermatogonial markers Lin28a (progenitors) or Kit (differentiating spermatogonia). Cl. 2 cells uniquely express the preleptotene marker Stra8, a key inducer of meiosis. Cl. 3 cells the meiosis specific Cyclin B3 (Ccnb3) gene, which is located on the $\mathrm{X}$ chromosome, marking the last stage where sex chromosomes are expressed (zygotene). Cl. 4 cells upregulate $\mathrm{H} 2 \mathrm{~A}$ histone family member $\mathrm{X}$ (H2afx), the histone variant associated with meiotic sex chromosome inactivation in early pachytene. Cl. $\mathbf{5}$ cells specifically express many genes related to the function of spermatids. e.g.: lactate dehydrogenase $C(L d h c)$, testis-specific enzyme in anaerobic glycolysis, producing energy in motile sperm.

Fig. 2: Multiplexed trajectory reconstruction is consistent with established expression patterns and allows to explore gene expression at high temporal resolution.

a.) Pseudotemporal ordering is consistent across 3 different reconstruction methods (min. pairwise $r>0.95$ ) and recapitulates the of stages (colors) defined earlier by marker genes. Each cell is a dot is a cell, ordered in 3 dimensions according to the pseudotime calculated by each algorithm (scaled 0-100) and colored as in Fig. 1.

b.) Consensus trajectory (color) is the average of the 3 methods and is represented on the tSNE map. Clusters are denoted by symbols.

c.) Three color single-molecule RNA FISH shows sequential expression of two consecutive prophase markers (Stra8, PI: Preleptotene; H2afx, P: Pachytene) and the Actb, specifically upregulated in post-meiotic cells. Roman numeral: spermatogenic stage; s116: step 1-16 spermatids; * marks the lumen and dashed line marks the base of the tubule; scale bar: $10 \mu \mathrm{m}$.

d.) Relative expression of autosomes (grey) and sex chromosomes (X: red, Y: orange) throughout prophase-I. While autosomes are steadily expressed, sex chromosomes are sharply downregulated before pachytene. Rolling average of normalized chromosomal read counts are scaled to by the median expression along the trajectory. The inset shows 
the raw transcript counts per cell as detected by sequencing, dropping before leptotene, and sharply increasing in pachytene.

e.) Expression of later stage marker genes from (Fig. 1) and other key genes resolve expression dynamics at a higher resolution. Early and late genes are separated in the top and bottom panel for clarity. Gene expression is displayed as z-score normalized rolling averages (window: 100). Temporal reconstruction precisely predicts expression timing. Lin28 peak is restricted to earliest Dazl+ spermatogonia, unlike Kit, and that the Stra8 slightly precedes $\operatorname{Prdm} 9$, as reported.

Fig. 3: Systematic analysis of expression timing and co-expression patterns finds novel co-expression patterns

a.) K-means clustering of the 1077 highly expressed genes identifies specific peaking time for each gene. Clustering was done on z-transformed moving averages of normalized gene expression.

b.) Traces of all genes in class 3 show a clear expression peak between 400 and 600 . The 3 sharpest peaking genes (by maximum z-score) are highlighted in color.

c.) Z-score normalized rolling average of raw transcript counts of all genes with a single, sharp peak, ordered by their peaking times. Note that the number of unique genes specific to the first (spermatogonia) and last (pachytene) cluster is inflated by nonspecific genes expressed beyond the analyzed time window.

d.) Search for similar expression dynamics identified multiple genes (e.g: Ube2t, Hdac1, Trim28) co-expressed with H2afx.

e.) $\mathrm{Gml}(\mathrm{Hemt} 3)$ vs $\mathrm{Gm} / 2$ (Hemt1) are the most stringently co-expressed genes with H2afx.

f.) Single-molecule RNA FISH shows the co-expression of $\mathrm{Gm} / 2$ and H2afx in Pachytene (P) spermatocytes in vivo. Roman numeral: spermatogenic stage; s1-16: step 1-16 spermatids; * marks the lumen and dashed line marks the base of the tubule; scale bar: $10 \mu \mathrm{m}$. WGA staining omitted for clarity.

g.) Single-molecule RNA FISH with wheat germ agglutinin staining (WGA, in yellow). WGA stains tubule boundaries and acrosomes, thus it allowed the staging of tubules in combination with nuclear morphology. Two adjacent tubules of different stages. The earliest pachytene cells are found in tubules of stage I-II, when pachytene cells just start to upregulate pachytene specific genes. Scale Bar: $10 \mu \mathrm{m}$

Fig. 4:. Meiotic entry is coupled to a downregulation of a tightly connected hub of transcription factors, and coincides with metabolic and transcriptional silencing.

a) Pairwise differential gene expression between spermatogonia (cl.1) and (pre)leptotene (cl.2) cells shows 113, and 109 genes at least 4-fold enriched in either cluster, at p.adj $<1 \mathrm{e}^{-6}$ (highlighted in red, Benjamini-Hochberg corrected). Selected gene names are displayed. All significant genes are listed in (Table.S4).

b) Semantic similarity map shows key, stage specific biological processes. Significant GOterms within the 4-fold spermatogonia (B), and (pre)leptotene (C) enriched genes -as identified in (A)- are grouped by similarity. Dot sizes correspond to the number of genes associated, colours denote the -log10(False Discovery Rate). Representative GO-terms with low FDR and high gene count are labeled. 
c) See above.

d) Z-score normalized expression heatmap of at least 2-fold, significantly up- or downregulated genes in spermatogonia or (pre)leptotene cells. Cells in each cluster are ordered along the trajectory. Selected representative genes are labeled.

e) List of 8 transcription factors with significantly enriched (p.adj. < 0.001) binding sites within spermatogonia specific 2-fold enriched genes, using the TRANSFAC and JASPAR databases.

f) The identified transcription factors form a densely connected protein-protein interaction network as identified by STRING.

g) Rolling average (window: 100) normalized gene expression of the detected transcription factors along the trajectory. Dashed lines indicate approximate spermatogonial and (pre)leptotene boundaries along the trajectory.

Fig. 5: A wave of sex chromosomal gene activation precedes meiotic sex chromosome inactivation (MSCl)

a.) $34 \%$ of sex chromosomal genes are induced specifically before and silenced during $\mathrm{MSCl}$. Genes are classified into 4 classes by k-means clustering of Z-score normalized rolling average gene expression profiles. Class 1 and 3 contains genes induced before and silenced by $\mathrm{MSCl}$. Class 2 contains spermatogonia specific genes, while class 4 contains leptotene specific genes, and genes which are steadily expressed until MSCI.

b.) Both sex chromosomes show increasing relative expression before a rapid silencing that coincides with the induction of $h 2 a f x$ gene. Rolling average normalized expression values are divided by 100 for $X$, or by 10 for the $Y$ chromosome for visualization. Dashed lines indicate the start and maximum of $h 2 a f x$ transcription, also marking the start and end of $\mathrm{MSCl}$. Pearson correlation coefficients between the sex chromosomes' and H2afx's expression during $\mathrm{MSCl}$ are denoted (X, $\mathrm{Y}$, respectively). Grey dashed line indicates transcript counts per cell, as in Fig.2D.

c.) Gene expression of known post-transcriptional regulators of H2afx. As reported Prkdc is not expressed and Atm is only expressed earlier, during double strand break formation. The sole activator expressed during $\mathrm{MSCl}$ is Atr, and its' expression is unchanged after (pre)leptotene, suggesting that $\mathrm{MSCl}$ may not only be controlled at the posttranscriptional level.

d.) Average expression of all peaking genes (class 1\&3) genes during $\mathrm{MSCl}$ (cl. 4) shows that a small set of genes is very highly expressed when meiotic silencing is already in progress.

e.) Individual traces of the highly expressed peaking genes (from D) shows a late and sharp silencing dynamics. H2afx expression (red), and $\mathrm{MSCl}$ boundaries (grey vertical lines) are displayed as a reference.

f.) Chromosomal location of the 27 sex chromosomal genes (all from chr. X) with an average expression above 1 in pachytene (cl.5).

g.) Highly expressed escapee genes. Average expression of sex chromosomal genes in pachytene II (cl.5) identifies a handful of escapee genes that are highly expressed after $\mathrm{MSCl}$. 
h.) The ampliconic gene Mageb16 is the only MSCl-escapee gene, which is also described to escape post-meiotic sex chromosome inactivation (PMSCI), and Car5b is the only gene that is also described as an escapee in female $X$ chromosome inactivation.

i.) Slitrk2 is the only gene whose expression anti-correlated with the total expression from the X-chromosome. While it is efficiently silenced before, it starts to be expressed during $\mathrm{MSCl}$ and increases throughout pachytene.

j.) Single-molecule RNA FISH shows that Slitrk2 escapes during late pachytene (right, stage IX) and it maintains post-meiotic expression (left) spermatids in vivo. Roman numeral: spermatogenic stage; Se: Sertoli cell; L: Leptotene; P: Pachytene; s1-16: step 1-16 spermatids; dashed line marks the base of the tubule; scale bar: $10 \mu \mathrm{m}$. WGA staining omitted for clarity.

k.) Single-molecule RNA FISH with WGA staining (in yellow) shows continued Slitrk2 expression in spermatids, but it is not expressed in the earliest pachytene cells (cl. 4). Scale Bar: $10 \mu \mathrm{m}$.

\section{Fig. 6: Genes in pachytene are induced in the same order as they are utilized in the} mature sperm.

a.) Differential gene expression analysis identifies 635 genes that are at least 3-fold upregulated in pachytene cells as compared to all other cells (p.adj $<1 e^{-6}$, in red, Benjamini-Hochberg corrected). Representative genes are labeled in black.

b.) Individual expression profiles of immediate genes show the step-like up regulation after $\mathrm{MSCl}$.

c.) The nuclear encoded mitochondrial gene, Cytochrome C Oxidase Subunit $8 \mathrm{C}(\mathrm{Cox} 8 \mathrm{c})$ is prime example of sharply upregulated genes.

d.) Gene expression profiles along the trajectory of the 635 genes were classified into 3 categories according to the shape and slope of increasing gene expression. K-means clustering was performed on z-transformed moving average gene expression.

e.) Gradual genes all showed a slowly increasing gene expression in pachytene.

f.) The uncharacterized Adam32 gene is suggested to be involved in sperm-egg fusion and it shows the typical gradual accumulation of fertilization related genes.

g.) Significantly enriched GO-terms suggest markedly different function between the immediate and gradual gene-sets (GO Biological Process and GO Cellular Component, $\mathrm{FDR}<1 \mathrm{e}^{-3}$ ). Immediate genes are enriched in energy and mobility related functions and structures, while the gradually upregulated genes are enriched in fertilization related functions and structures.

h.) Gradual- and immediate-class specific GO-terms map to different anatomical regions of the sperm. Both unique and shared enriched GO-terms are analyzed, and informative GO-terms are displayed. Gradual-class specific terms and corresponding anatomical structures are in red. Immediate-class specific terms and structures are in green. Shared terms and the related axoneme is indicated by the black arrow. The full list of GO-terms is accessible in (Table S6). 
i.) Single-molecule RNA FISH shows the upregulation of the fertility related Adam32 after pachytene and its post-meiotic expression in spermatids in vivo. Roman numeral: spermatogenic stage; B: B spermatogonia; P: Pachytene spermatocyte; s1-16: step 1-16 spermatids; * marks the lumen and dashed line marks the base of the tubule; WGA staining for acrosomes is in yellow; scale bar: $10 \mu \mathrm{m}$.

\section{Supplementary figure legends}

\section{Fig. S1. Filtering and quality control for clustering}

-(A) Transcript count per cell shows a bimodal distribution. Low quality cells below 3000 transcripts are discarded (red). This results in 1274 high quality cells (green) with a median of 20488 reads.

-(B) Saturation determines the optimal cluster number in RacelD2. With increasing number of clusters, the Within Cluster Dispersion (WDC) decreases. When further increase of the number of clusters (k) yields a decrease (grey) that is within the estimated error interval (red) of the decrease, $\mathbf{k}$ is selected ( 5 in this case).

-(C) Gap Statistic did not yield a peak in single cell mRNA-seq datasets, therefore cannot be used to unambiguously determine the cluster number. Of not that it also saturates around 5 , the cluster number determined by the saturation of within cluster dispersion.

-(D) The silhouette plots showed the average similarity of each cell to all cells in its own cluster, relative to other cells in the closest neighboring clusters. Positive values for most cells suggest that they are well represented within their own cluster.

-(E) High Jaccard index shows the high reproducibility of clusters across multiple bootstrapping rounds. The Jaccard index is calculated as the intersect over the union of cells in the same cluster across multiple bootstrapping rounds, pairwise. RacelD qualifies clusters reproducible above Jaccard index $60 \%$ (or 0.6 ).

-(F) Sequencing depth does not explain observed transcript count differences. Average over-sequencing per cell varies across individual cells a magnitude more than across clusters. Average oversequencing per cluster is lower in clusters with high transcript counts ( $\mathrm{cl} 1 \& 5)$ as expected if the high read counts were explained by biology, and not by sequencing depth.

\section{Fig. S2. Differences in transcriptome and clustering results are not explained by technical} effects.

-(A) Distribution of cells across cell types, which were identified by k-medoids clustering.

-(B-D) Cells from all four libraries (B), all three animals (C), and cells prepared with either CEL-Seq1 or CEL-Seq2 primers (D) -- denoted by color, respectively -- contribute equally to all cell types (denoted by shapes), as shown on the t-SNE map. 
-(E-F) Detected Gene and Transcript counts per cell detected both transiently decrease from meiotic entry $(\mathrm{cl} .1 \rightarrow \mathrm{cl} .2)$ until a global transcriptional activation in pachytene (cl.5).

\section{Fig. S3. Pseudotemporal ordering by three different algorithms}

-(A) Principal graph of cluster centers, and cell projections as reconstructed by Monocle 2 using DDRTree algorithm. Colors denote cell clusters as determined by RacelD, colored by Monocle2.

-(B) Minimum spanning tree of cluster centers, as reconstructed by TSCAN (in black). Cells were subsequently projected on this tree. Colors denote cell clusters identified by TSCAN using a multivariate Gaussian mixture model in PCA space.

-(C) Reconstructed Principal Curve in t-SNE space and minimal cell projections, to the curve. Colors denote cell clusters as determined by RacelD. Clustering results are not used in this reconstruction, only provided for easier comparison.

-(D) Pairwise scatterplots and Pearson correlation coefficients across the 3 reconstruction methods.

\section{Fig. S4. Filtering Peaking Genes by Quantile Ratio}

-(A) The ratio of the $100 \%$ and $95 \%$ quantiles of each gene's expression efficiently distinguishes peaking genes (high maximal z-score) caused either by outlier expression values, or by representative gene expression. While these genes both have high z-score maxima (31 and 17 respectively), quantile ratio of two example genes clearly separates outlier genes like Prm1. Log quantile ratio shown by dotted lines for Zfy2 (left) and Prm1 (right).

-(B-C) Normalized transcript counts in single cells and rolling averages (in black, window: 50) of Prm1 and Zfy2. Maximum z-scores of rolling averages are high in both cases, however in the case of Prm1, this is clearly caused by one outlier cell with 1000 transcripts, thus it does not reflect a general change in expression.

-(D) Entropy quantifies the diversity of the transcriptome per cell and showed that pachytene-Il cells (cl.5) had transcriptome diversity even higher than spermatogonial stem cells.

\section{Fig. S5. Expression patterns during and after $\mathrm{MSCl}$ in cluster 4 \& 5}

- (A) Correlation network of gene expression plotted in PCA space. Spearman correlation was calculated on the normalized expression patterns of sex chromosomal genes along the trajectory within cluster $4 \& 5$. The blue arches represent high Spearman correlation coefficients. All parameters were left as default in the corrr R-library. Most genes are like Ndufa1 (big cluster on the left), in that their normalized expression (relative to the total transcript count per cell) decreases, while absolute transcript count might stay at a similar level. Sporadically, escapee genes show particular expression 

patterns, such as genes similar to Ptchd1, showing flat relative, increasing absolute counts, or a strong increase such as Slitrk2.

-(B) The double peaking expression pattern of the multicopy Rbmy gene family.

\section{Fig. S6. Changes in molecular chaperones' expression coincides with major transcriptional changes, (activation and silencing).}

-(A) Constitutively expressed (housekeeping) Hsp90ab1 (90a-class b) and Hspa8 expression decreases upon meiotic entry, alongside with 3 chaperones involved in protein import to mitochondria (Hspd1, Hspe1, and Hspa4)

-(B) From (pre)leptotene to zygotene a mixed set of chaperones is induced. The constitutive Hsp90b1 shows moderate induction ( 20-30 mRNA/cell), while the endoplasmic reticulum related, inducible Hspa5 shows very strong upregulation ( 70$80 \mathrm{mRNA} / \mathrm{cell})$. The signaling related small HSP member Hspb11 shows a minor ( 5mRNA/cell), but distinct peak at meiotic entry.

-(C) The induced Hsp90a is known to be involved in cell cycle regulation, and it is strongly induced upon meiotic entry, reaching $\sim 150$ transcripts per cell. Beside its role in stress response, $H s p 90 \alpha$ is also induced in response to the induction of excessive translation. At the same time moderate to low induction of small HSPs Hspb6 and $H s p a 4 I$, and the Hsp70 family member Hspa2, which is suggested to be involved in spindle integrity.

-(D) Log10 scaled moving average gene expression of all displayed chaperones. Importantly, immediate early genes (as defined in van den Brink et al 2017) are mostly lowly expressed in the dataset, or specifically expressed at different stages (see also at Data S5).

-(E) Expression of Mid1 (Fxy), the only detected genes from the pseudoautosomal region (PAR). 


\section{Supplementary table descriptions}

\section{Table S1: Differential gene expression: each cluster vs. all others}

Differential gene expression analysis of each cluster as compared to all other clusters. Summary for each cluster (number of genes enriched), summary for each gene (arithmetic and geometric mean expression per cluster) and the enrichment statistic per gene, per comparison are

1003 provided.

\section{Table S2: Differential gene expression: stepwise comparisons}

Differential gene expression analysis of each cluster compared to the previous (in developmental order) cluster. Summary for each cluster (number of genes enriched), summary for each gene (arithmetic and geometric mean expression per cluster) and the enrichment statistic per gene, per comparison are provided.

Table S3: Changes of gene expression and function during meiotic entry.

TRANSFAC and JASPAR enrichment of transcription factors, binding to all at least 2-fold enriched genes; enrichment statistic per gene, per comparison; and the list of significantly enriched GO-terms in gene products of cluster 1 and 2 cells are provided.

Table S4: Changes of gene expression and function during meiotic sex chromosome inactivation

Characteristic silencing time and peak gene expression (rolling average) of sex chromosomal genes during $\mathrm{MSCl}$; List of early-silenced genes and respective significantly enriched GO-terms; the same information for late-silenced genes; and finally, the list of sex chromosomal genes, which were used as a statistical background for the enrichment analysis. products for each cluster; and respective lists of enriched GO-terms are provided. 


\title{
Supplementary dataset descriptions
}

\section{Data S1 Transcriptome wide clustering as well as marker gene expression supports gradual, as opposed to switch-like differentiation of spermatogonia}

\begin{abstract}
A. Dazl+ spermatogonial transcriptome does not sub-cluster along Kit or Lin28a/Sox13/Plzf expression boundaries. Spearman or Pearson correlation based clustering of spermatogonia confirms overall transcriptional similarity observed in Fig. 1 regardless of the expression of Kit or the markers of undifferentiated spermatogonia. The cells that cluster apart are of lower quality, as defined by raw transcript counts.
\end{abstract}

B. Expression of reported markers of Kit-, undifferentiated spermatogonia. All spermatogonial cells exclusively express Dmrt $1.77 \%$ of spermatogonia express Kit ( $\geq 1$ raw transcript), and only these cells also express Cyclins (Ccnd1, Ccna2, Ccng1). Kit-Dazl+ (and Dmrt1+) spermatogonia exclusively express a set of markers for undifferentiated spermatogonia: Plzf (or Zbtb16), Sall4, and Sox13. However, the expression of other markers for undifferentiated spermatogonia, such as: Uchl1, Sox3, Stella (Dpp3), or Lin28a), are not restricted to the Kitpopulation.

\section{Data S3 Differential Gene Expression}

Visual summary of differential gene expression analysis (Table S1-S2). Panels 1-5: comparison of cells in each cluster with all other cells. Panels 6-9: comparison of each cluster with the developmentally earlier cluster. Genes in red are significantly differentially expressed at $p<1 e^{-6}$ (Benjamini-Hochberg corrected).

\section{Data S5 Stress.Genes.van.den.Brink.2017}

Dissociation-induced genes (covering most early immediate genes) as defined in (Van Den Brink et al., 2017), are mostly lowly expressed. Expressed "dissociation-induced genes" show varied dynamics along prophase-l and do not point to population of cells, which is particularly affected by the isolation procedure. 


\section{Supplementary online methods Vertesy et al}

1051 Mice strains, Organ Isolation and Dispersion

1052 To separate germ cells from somatic cells, as well as to selectively sort germ cells from 1053 meiotic prophase-I, we used a mouse line expressing Dazl-GFP-V5 on a bacterial 1054 artificial chromosome (BAC). In this construct, the GFP sequence is integrated directly 1055 before the stop codon, and its expression is controlled by Dazl promoter and enhancer 1056 sequences (Chen et al., 2014). All mice were housed according to the Hubrecht Institute 1057 guidelines, which include access to food and water ad libitum. All animal experiments were carried out in compliance with standards for care and use of laboratory animals, with approval from the Dutch Animal Experiment Committee of The Royal Netherlands Academy of Arts and Sciences (KNAW). Mice were between 2 and 3 months old at time of sacrifice.

In the first step, we isolated testicles from Dazl-GFP mice and chopped them into pieces. Next, these tissue pieces were transferred to a $50 \mathrm{~mL}$ falcon tube and incubated in a collagenase type II (2 mg/mL Gibco, 17101015)/phosphate-buffered saline (PBS, Lonza, 17-516F) solution placed in a water bath set at $37^{\circ} \mathrm{C}$ for 1 hour to degrade the extracellular matrix and release cells from their niche. We used $10 \mathrm{~mL}$ collagenase solution per TA. After this initial incubation, the tissue pieces were incubated in Trypsin at 37 degrees with gentle intermittent shaking for 8-10 min until the islets were dispersed into single cells. The dispersed tissue was washed briefly with cold PBS then filtered through a $25 \mu \mathrm{m}$ sieve to remove debris and aggregates.

\section{FACS Sorting}

1072 The Dazl-GFP mice allows us to select germ cells from spermatogonial up to pachytene spermatocyte stages, as Dazl expression in adult testes is restricted to germ cells of 1074 these stages. We used Propidium lodide (PI) to exclude dead cells and VybrantViolet 1075 (cat. V35003) staining for DNA content to exclude haploid cells.

1076 We incubated the cells with PI and VybrantViolet on ice before FACS-sorting, according 1077 to manufacturer's protocol. Cells were kept on ice until sorting. We used FACSJazz (BD 1078 biosciences) with the following configuration for all sorts Channel, Dye, Function: [488] 1079 530/40, GFP, Dazl expression; [488] 585/29, PI, Live cells (PI negative); [405] 450/50, 
1080 VybrantViolet, DNA content. Channel interpretation: [Excitation wavelength] Emission 1081 wavelength / Detection bandwidth.

1082 First, we selected single, live cells based on PI exclusion and scattering (P1 and P2 and 1083 P3). Next, we used GFP expression and VybrantViolet staining of Live Dazl-GFP 1084 positive single-cells. Finally, the selected cells were sorted into hard shell 384-well 1085 plates (Bio Rad, cat HSP3801) containing $200 \mathrm{nl}$ of RT primers, dNTPs and synthetic 1086 mRNA Spike-Ins. To avoid evaporation, $5 \mu$ l of vapor-lock (QIAGEN, cat 981611) was 1087 added on top.

1088 After sorting, we spun down and froze the plates immediately. The plates were stored at $-80^{\circ} \mathrm{C}$ until processing. For details see (Van Den Brink et al., 2017). For library preparation and sequencing we followed the SORT-seq protocol (Muraro et al., 2016). Sorting experiments were done in biological triplicate and technical replicates. There were no relevant differences between replicates that would affect the conclusions. We both used CEL-seq1 and CEL-seq2 based primers. They showed differential sensitivity for a set of genes, which we effect we normalized out. See Analysis for consequences and normalization.

\section{Single-Cell mRNA library preparation}

1097 We prepared cells into sequencing libraries by SORT-seq. For the detailed protocol, see: (Muraro et al., 2016) and (Van Den Brink et al., 2017). In brief, we used a 3-prime, poly-A based, UMI-corrected library construction protocol. SORT-seq is an improved and robotized version of the CEL-seq2 protocol designed for FACS sorted cells (Hashimshony et al., 2016).

Microscopy, single-molecule RNA fluorescence in situ hybridization (smFISH).

For microscopy, testis isolated from 2-3 months old male wild type mouse were fixed in 4\% RNAse free PFA at room temperature for 4 hours and cryopreserved by incubating overnight in $30 \%$ glucose solution at $4 \mathrm{C}$. Tissue was then cryomounted on OCT , frozen and sectioned into $10 \mu \mathrm{m}$ thick slices. Tissue sections were then used for performing single-molecule RNA-FISH using the ViewRNA ISH Cell assay kit (Invitrogen: QVC0001). Tissue sections undergo a new fixation with 4\% RNAse free PFA for $20 \mathrm{~min}$ at RT, permeabilization and protease treatment steps. Next, gene specific probe sets are hybridized ( 20 oligos per gene), followed by 
sequential branched-DNA (bDNA) signal amplification, and finally individual mRNA molecules are visualized. The probes were designed to hybridize with the mRNAs of Stra8 (VB6-16903VC), H2afx (VB1-3030165-VC), Actb (VB4-10432-VC), Gml2 (VB6-3223290-VC), Slirtk2 (VB63218124-VC), Adam32 (VB6-3221253-VC). Nuclei were stained by Hoechst, lectins were stained by Wheat Germ Agglutinin (WGA, molecular probes \#W11261). Sections were imaged using a 63x objective on a PerkinElmer Ultraview VoX spinning disk microscope combined with a Leica SP8. Images were analyzed in ImageJ/FIJI.

\section{Histology and staging of seminiferous tubules}

1120 Seminiferous tubules a classified into 12 stages (I-XII) based the presence of different cell types (Russell et al., 1993). Many cell types are indistinguishable by themselves under the microscope but can be inferred if the stage of the tubule is known, because only certain cell types are present at each tubule stage. Staging of tubules is based on nuclear and acrosomal morphology, as well as the position and presence of certain cells types (Meistrich and Hess, 2013). We staged tubules by fluorescence microscopy using a combination of fluorescent lectin conjugates and nuclear morphology (Nakata et al., 2015). Stages are indicated by Roman numerals. Each image was recorded in 4 fluorescent channels, either DAPI $+3 \mathrm{smFISH}$ probes, or DAPI + WGA +2 smFISH probes. For clarity, DAPI + smFISH images are shown in the main figures, while 1129 WGA staining is not always shown.

\section{Alignment and Quantification}

1132 Paired-end reads from Illumina sequencing were aligned to the mouse RefSeq transcriptome with BWA-MEM (Li, 2013). We trimmed low quality bases and discarded

1134 low quality and ambiguous alignments. For details on alignment, see (Grün et al., 2015).

1135 After alignment, we removed UMI-duplicates from uniquely mapping reads. UMI-

1136 duplicates are reads from the same cell, same gene sharing the same unique molecular 1137 identifier revealing that they are were amplified from the same original mRNA molecule.

1138 Next, based on binomial statistics, we converted the number of observed UMI-s into 1139 transcript counts (Grün, Kester and van Oudenaarden, 2014). 
1141 First, we selected cells with >2000 transcripts, yielding 1274 high quality cells. Next, we 1142 selected genes with at least 2 transcripts in at least 20 (out of 1274) cells. These cells 1143 had on average a median of 8079 transcripts from 1077 highly expressed genes.

\section{Downsampling and normalization}

1145 Samples were normalized by taking the average of 10 downsampling rounds. Generally speaking, downsampling is better at keeping the statistical properties of the data, such as noise level, while normalization has no loss of information and therefore can be more sensitive. We combined these two approaches for an optimum solution. For downstream analysis, including k-medoids clustering, we used RacelD (Grün et al., 2016) and custom scripts.

1151 Normalization of libraries generated by CEL-seq1 and CEL-seq2 primers

1152 As the SORT-seq protocol was designed during the time course of this study, we 1153 prepared libraries with two types of primers for reverse transcription. The original version 1154 is described in (Grün, Kester and van Oudenaarden, 2014), and it was based on CEL1155 seq1 (cs1) (Hashimshony et al., 2012). Later, an optimized primer version was developed (Muraro et al., 2016), which was based on the CEL-seq2 (cs2) protocol

1157 (Hashimshony et al., 2016). We prepared 490 high quality (filtered) cells by cs1-, and 1158766 by cs2-primers.

1159 To normalize the 2 protocols, first we calculated the average expression per gene over 1160 all index sorted plates per cs1 and cs2 batches. Next, we calculated the ratio of the 1161 batch averages, and normalized all genes to have the same average expression in both 1162 batches. After normalization we filtered highly expressed genes which are then used for 1163 clustering. Genes which had a more than 10-fold bias before normalization or were 4 1164 times more often detected in either protocols were excluded from clustering. In Fig. S2 we show that cells from each batch contribute equally to all clusters regardless of primer design, biological replicate, or batch of library preparation.

\section{Clustering and tSNE}

1168 For many steps of analysis, including k-medoids clustering of cells and tSNE projection, 1169 we used an in-house improved version of RacelD2 (Grün et al., 2016). We implemented 1170 parallel computing in RacelD2 and extended its functionality to handle pseudotime 1171 related analysis and better accommodate multi-panel plotting. The code will be freely 
1172 available on https://github.com/vertesy/RacelD-v2.5-Multithreaded upon acceptance,

1173 with restriction to attribute the original author of RaceID upon use.

1174 Establishing the number of clusters for k-medoids clustering

1175 In RacelD, the optimal cluster number is determined as the saturation point of the Within

1176 Cluster Dispersion (WDC). By separating the data into more (thus smaller) clusters

1177 WDC decreases. When adding a new cluster yields a decrease that is no bigger than

1178 the estimated error interval of the decrease, $\mathrm{k}$ is selected. In this estimation, we

1179 repeatedly clustered cells into 1 to 10 preset clusters, and calculated WDC for each

1180 number of clusters. The optimal cluster number was estimated to be 5.

1181 For comprehensiveness, we also calculated gap statistics (Tibshirani, Walther and 1182 Hastie, 2001). Gap statistic in single cell mRNA-seq datasets often does not yield a 1183 peak, therefore cannot determine the cluster number unambiguously. Nevertheless, it 1184 also saturates around 5, as estimated before

1185 The silhouette plot shows the average similarity of each cell to all cells in its own cluster 1186 relative to all cells in the closest neighboring clusters. Positive values for most cells 1187 suggest that they are well represented within their own cluster. High Jaccard index 1188 shows high reproducibility of clusters across multiple bootstrapping rounds. The Jaccard 1189 index is calculated as the intersect over the union of cells in the same cluster across two 1190 bootstrapping rounds. RaceID qualifies clusters reproducible above Jaccard index $60 \%$, 1191 while Jaccard indices in this study range $74-99 \%$.

\section{Differential Gene Expression}

1193 For differential gene expression analysis, we used RacelD2. The algorithm was specifically designed to model transcript count variability in single-cell data by a negative binomial noise model (Grün, Kester and van Oudenaarden, 2014), building on (Anders 1196 and Huber, 2010). P-values of significantly up or downregulated genes were calculated 1197 based on Poissonian statistics and corrected for multiple testing by the Benjamini1198 Hochberg method (Grün and van Oudenaarden, 2015).

1199 We found that fold change as commonly calculated as the fraction of arithmetic mean 1200 ("average") gene expression in compared cell types is not well suited for single cell data, 1201 as suggested previously (Bengtsson et al., 2005). 
1202 Analysis based on arithmetic mean often identifies genes, which we consider false 1203 positives when looking at the raw expression data, in single cells. This is because 1204 arithmetic mean is a metric sensitive to outliers. It often indicates a big differences, 1205 which are not representative of the group of cells; in fact they are regularly caused by a 1206 few outlier cells with very high expression.

1207 Median is commonly used as an outlier robust metric of the central tendency, it however 1208 loses sensitivity at low integer read counts, which is the typical range the for majority of 1209 the genes in single cell data. Furthermore, median becomes meaningless if near $50 \%$ of 1210 the cells have 0 expression (also known as zero-inflated data). Subtype specific genes 1211 with a narrow expression window are often overlooked by using median (e.g.: Zfy1 \& 2).

1212 Geometric mean is both robust against outliers and handles zero-inflated data, and it 1213 was found to be the appropriate metric for gene expression data that varies on the log1214 scale (Vandesompele et al., 2002; Bengtsson et al., 2005; Goymer, 2005). We therefore 1215 used the fold change of geometric means alongside the classical definition of fold 1216 change of arithmetic means, so that a gene must satisfy the same threshold for both 1217 metrics. This largely decreased our false positive detection, while maintaining similar 1218 sensitivity.

1219 Expression patterns defining cluster boundaries

1220 Groups of cells can be defined by either a unique expression of genes or by a combination of multiple non-unique expression patterns. We compared gene expression in each cluster to all other clusters to find uniquely expressed genes (TableS2), and we also compared gene expression in each cluster to the previous cluster (=developmental stage) to find gradual or stepwise changing genes (Table.S3).

We found that GO-term enrichment on coding genes gives cleaner results, therefore we calculated GO-term enrichments using the STRING (v10.5) protein interaction database (Mering, 2003) everywhere, unless stated otherwise.

Functional enrichment and REViGO Analysis for spermatogonial and (pre)leptotene genes

1231 We first performed pairwise differential gene expression between spermatogonial and 1232 (pre)leptotene clusters, then calculated GO-term enrichment on 4-fold enriched genes in either clusters. Next we analyzed the significantly enriched GO-terms (Biological Process, FDR < 0.05) 
using REViGO (Supek et al., 2011), with setting (similarity=0.9, semantic similarity measure: SimRel, associated values: -log10(FDR), GO term sizes: Mus musculus). REViGO first removes redundant (nested) GO terms, then calculates a 2 dimensional multidimensional scaling map based on semantic similarity of the terms. The full list of GO-terms is provided in (Table S3).

\section{GO annotation defined gene sets}

We first identified relevant GO-terms using EBl's QuickGo (Binns et al., 2009) within the GOterm hierarchy. Next, we downloaded mouse gene annotations for each term, excluding negative annotation qualifiers using the AmiGO browser (Carbon et al., 2009). We selected genes detected in our dataset and analyzed genes as described per analysis.

\section{Pseudotemporal ordering of single cells}

1244 To draw a comprehensive picture of all genes changing expression at different times, we needed to reconstruct their developmental trajectory (Cannoodt, Saelens and Saeys, 2016). As we did not measure time, but infer, how long cells progressed in spermatogenesis, this procedure is called pseudotemporal ordering. We ordered cells according to gradual, transcriptome wide changes in gene expression by 3 , distincts and

1249 established methods: Monocle 2 (Qiu et al., 2017), TSCAN (Ji and Ji, 2016) and 1250 principal curve (Hastie and Stuetzle, 1989) as used in (Petropoulos et al., 2016). The 3 1251 methods are compared in their main features, dimensionality reduction, clustering, and trajectory modeling below.

\begin{tabular}{|c|c|c|c|c|}
\hline Approach & $\begin{array}{l}\text { Dimensionality } \\
\text { Reduction }\end{array}$ & Clustering & Trajectory model & Ref \\
\hline $\begin{array}{l}\text { Monocle } \\
2\end{array}$ & $\begin{array}{l}\text { DDRTree } \\
\text { (discriminative } \\
\text { dimensionality } \\
\text { reduction via } \\
\text { learning a tree) }\end{array}$ & $\begin{array}{l}\text { k-medoids, } \\
\text { provided by } \\
\text { RaceID2 }\end{array}$ & $\begin{array}{l}\text { Principal Graph (a } \\
\text { Principal Curve with } \\
\text { branches allowed) } \\
\text { learnt during } \\
\text { dimensionality } \\
\text { reduction by DDRTree. }\end{array}$ & $\begin{array}{l}\text { (Qiu et al., } \\
\text { 2017) }\end{array}$ \\
\hline
\end{tabular}




\begin{tabular}{|c|c|c|c|c|}
\hline TSCAN & $\begin{array}{l}\text { PCA (Principal } \\
\text { Component } \\
\text { Analysis) }\end{array}$ & $\begin{array}{l}\text { Multivariate } \\
\text { gaussian } \\
\text { mixture in } \\
\text { PCA space }\end{array}$ & $\begin{array}{l}\text { Minimum spanning tree } \\
\text { of cluster centers }\end{array}$ & $\begin{array}{l}\text { (Ji and Ji, } \\
2016)\end{array}$ \\
\hline $\begin{array}{l}\text { Principal } \\
\text { Curve }\end{array}$ & $\begin{array}{l}\text { tSNE ( } t- \\
\text { distributed } \\
\text { stochastic } \\
\text { neighbor } \\
\text { embedding) }\end{array}$ & None & Principal Curve & $\begin{array}{l}\text { (Hastie and } \\
\text { Stuetzle, } \\
\text { 1989) }\end{array}$ \\
\hline
\end{tabular}

\section{Monocle 2}

Monocle 2 identifies differentially expressed genes across clusters of cells (provided reconstruct a minimum spanning tree connecting cluster centroids, which is called principal tree. Cells then are projected to this principal tree, and their position along the backbone of the tree is counted as pseudo time. Monocle 2 uses an unsupervised inference method, where one does not need to feed the number of trajectories or paths, nor branching points. In fact multiple minor (<8\% of the cells) side branches are detected by the algorithm, which all fall close to the main tree (Fig. S3).

\section{TSCAN}

In TSCAN, we provide the filtered normalized dataset. TSCAN uses PCA to reduce the

1268 dimensionality, which is followed by clustering by fitting a mixture of multivariate normal 1269 distributions using the Mclust package. The number of clusters here is determined by 1270 Bayesian information criterion, in our case it was 5. Next, TSCAN connects cluster centers with a minimum spanning tree, and finally it projects single cells on the tree, which is the cell order (Fig. S3 B). 


\section{Principal Curve}

1274 We fitted a principal curve (Hastie and Stuetzle, 1989), as in (Petropoulos et al., 2016) to 1275 the two-dimensional t-SNE map (Fig. 1 A) of the cells in 100 iterations. The curve is 1276 initialized as a line segment; we use first principal component line. Next, each point of 1277 the curve is recalculated as a local average of all data points that project to this points using a spline smoothing with 4 degrees of freedom (smooth.spline function). In the next round new projections are calculated, etc. Already after 10 iterations the curve converged. The final curve was calculated after 100 iterations (Fig. S3 C).

Comparison and Consensus or Multiplex Pseudotime

1282 Projection coordinates in all 3 approaches are scaled to $[0,100]$ and pairwise Pearson 1283 correlation coefficients are calculated: TSCAN vs. Principal Curve: 0.956; TSCAN vs. 1284 Monocle: 0.973; Principal Curve vs. TSCAN: 0.956. Essentially all 3 algorithms captured 1285 the linear progress from spermatogonia to pachytene spermatocytes (Fig. 2 C). To 1286 further increase robustness, we calculated the consensus, or multiplex pseudotime as 1287 the average pseudotime for each cell in all three trajectories calculated by the different 1288 algorithms //Table.S3.

co-expression analysis

We calculated the Pearson correlation coefficient ( $r$ ) over the normalized gene 1291 expression of pseudotime ordered cells.

1292 Gml (Hemt3) and Gml2 (Hemt1)

$1293 \mathrm{Gml}$ (Hemt3) and $\mathrm{Gm} / 2$ (Hemt1) is an interesting pair of genes, which are encoded on 1294 opposite strands, 250 bases apart (ensembl mouse, GRCm38.p6, Chromosome 15: $129574,806,933-74,812,856)$. While only $\mathrm{Gml}$, but not $\mathrm{Gml} 2$ protein was shown to be expressed in spermatocytes in prophase (Xue et al., 1999), the two transcripts show $89 \%$ sequence identity, as determined by blast alignment of the cDNAs. Sequencing reads show roughly equal and high read counts for both genes, suggesting that this readout is not due to sequencing errors (in such a case, one transcript would have 1-

$130010 \%$ of the reads from the more abundant transcript).

1301 Both transcripts are extremely well correlated with H2afx along pseudotime (r1 r2 =??), 1302 and accordingly, the $\mathrm{Gml}$ signal is correlated with H2afx signal in SmFISH. However, 1303 because of the sequence similarity, it is not possible to resolve $\mathrm{Gml}$ and $\mathrm{Gm} / 2$ by 
1304 smFISH (transcripts are tiled along with 20+ oligos), therefore smFISH gives a readout 1305 of these transcripts together.

\section{Early- and late-silenced genes on the sex chromosomes}

First, we selected all sex chromosomal genes that had a running median expression maximum above 1 during $\mathrm{MSCl}$ (pseudo time points 650-900) as defined by the h2afx expression ramp (Fig. 5B). To quantify the pace of gene expression changes, we defined the characteristic timescale of silencing (Ts) for each gene. Ts is defined in a way similarly to a substance's half-life: it is the time (pseudo time points) required for gene expression to drop to half of its maximal value, and it was corrected with an offset if the maxima did not coincide with beginning of $\mathrm{MSCl}$. Without this correction, late activated genes, e.g. Scm/2, would be mistakenly counted as an early-silenced gene. Ts was calculated on rolling average normalized gene expression with a window of 100 . Finally, we ordered all genes according to their characteristic silencing timescale and divided the distribution into earlier and later half. We note that some reads mapped to a microRNA, Mir684-1. It is a multicopy gene across autosomes and the $\mathrm{X}$ chromosome. For this reason, and since CEL-seq is not suited to study microRNAs, we considered it a mapping artifact, therefore we excluded it from the analysis.

GO-term enrichment was calculated on the interaction network of coding genes as described above. The statistical background for the enrichment were all coding genes on sex chromosomes present in the gene model used (mm10), (Grün, Kester and van Oudenaarden, 2014).

\section{Escapees}

As pachytene cells had $\sim 9$ fold more reads than zygotene, relative expression levels might not be indicative of leaky silencing, therefore we calculated expression of unnormalized transcript counts. The variation among single cells was counteracted by calculating a rolling average over the cells ordered along the trajectory.

\section{Classification gene expression patterns along the trajectory}

First, the normalized gene expression values of individual cells were ordered along the trajectory. Next, we calculated the rolling average expression with a window of 50 (or 100 when indicated). To make genes with widely different expression levels comparable 
1334 regarding their activation or silencing time, we z-score transformed these values. Finally,

1335 this gene expression matrix was classified by k-mean clustering.

1336 Transcriptome wide identification of time-specific or peak genes

1337 As we extended our analysis to lowly expressed, we found many lowly expressed genes 1338 with specific expression times, such as Zfy2 (Fig. S3 C). Expression peaks in ordered 1339 series can be identified by maximum z-score, however single outliers cause artifactual 1340 high z-scores (Fig. S3 B), but in this expression regime, one cannot filter on expression 1341 level. Therefore, we calculated the quantile-ratio, a metric that can robustly remove 1342 artifactual peaks. In this, we calculate the ratio of the $100 \%$ and $95 \%$ quantiles of each 1343 gene's expression. Genes only highly expressed in a few outlier cells have extremely 1344 high quantile ratio, and this metric efficiently distinguishes these genes from genes with 1345 true expression peaks (Fig. S3 A).

\section{Analysis of Transcription Factor Binding sites}

1347 We found that meiotic entry from the spermatogonia towards preleptotene is 1348 characterized by global transcriptional silencing accompanied by the upregulation of 1349 defined set of genes. To find out which transcription factors driving these changes, we 1350 analyzed transcription factor binding sites enriched in the all genes that are at least 21351 fold differentially expressed in cluster 2 vs 1 , and vice versa. We used the online too 1352 Enrichr (Chen et al., 2013; Kuleshov et al., 2016), which searches across the 1353 TRANSFAC and JASPAR transcription factor binding profiles (Matys, 2006; Portales-

1354 Casamar et al., 2010). Enrichr scans the -2000 and +500 vicinity of transcription start 1355 sites for transcription factor binding sites. The list of transcription factors with

1356 significantly enriched binding sites is then analyzed in the 2017 version of the amazing 1357 STRING database to identify functional and physical interactions, as well as GO-term enrichment 1358 (Mering, 2003; Szklarczyk et al., 2017). We found that all upregulated genes are regulated by a 1359 tightly interconnected set of transcription factors. 
1362 All analysis was performed in R-studio v1.0.136. For single cell analysis we used the 1363 StemID algorithm (Grün et al., 2016) and custom scripts. Figures were generated by R 1364 packages pheatmap v1.0.8 (Raivo, 2013) and MarkdownReports v2.9.5 (Vertesy, 2017). 1365 Upon acceptance, the source code for analysis will be available "as-is" under GNU 1366 GPLv3 at https://github.com/vertesy/Spermatogenesis.Single-Cell. Sequencing data is 1367 deposited under accession number GSE114788 at GEO.

Adams, S. R. et al. (2018) 'RNF8 and SCML2 cooperate to regulate ubiquitination and H3K27 acetylation for escape gene activation on the sex chromosomes', PLoS Genetics, 14(2). doi: 10.1371/journal.pgen.1007233.

Anders, S. and Huber, W. (2010) 'Differential expression analysis for sequence count data', Genome biology, 11(R106), pp. 1-12. doi: 10.1186/gb-2010-11-10-r106.

Anderson, E. L. et al. (2008) 'Stra8 and its inducer, retinoic acid, regulate meiotic initiation in both spermatogenesis and oogenesis in mice', Proceedings of the National Academy of Sciences, 105(39), pp. 14976-14980. doi: 10.1073/pnas.0807297105.

Aravin, A. a, Hannon, G. J. and Brennecke, J. (2007) 'The Piwi-piRNA pathway provides an adaptive defense in the transposon arms race.', Science (New York, N.Y.), 318(5851), pp. 7614. doi: 10.1126/science.1146484.

Baarends, W. M. et al. (2005) 'Silencing of unpaired chromatin and histone H2A ubiquitination in mammalian meiosis.', Molecular and cellular biology, 25(3), pp. 1041-53. doi: 10.1128/MCB.25.3.1041-1053.2005.

Bellani, M. A. et al. (2005) 'SPO11 is required for sex-body formation, and Spo11 heterozygosity rescues the prophase arrest of Atm-/- spermatocytes.', Journal of cell science, 118(Pt 15), pp. 3233-45. doi: 10.1242/jcs.02466.

Bengtsson, M. et al. (2005) 'Gene expression profiling in single cells from the pancreatic islets of doi: $10.1101 /$ gr.3820805.4.

Berletch, J. B. et al. (2015) 'Escape from X inactivation varies in mouse tissues.', PLoS genetics. Edited by M. S. Bartolomei, 11(3), p. e1005079. doi: 10.1371/journal.pgen.1005079.

Bhattacharyya, T. et al. (2014) 'X Chromosome Control of Meiotic Chromosome Synapsis in Mouse Inter-Subspecific Hybrids', PLoS Genetics. Edited by B. A. Payseur, 10(2), p. e1004088. doi: 10.1371/journal.pgen.1004088. 10.1093/bioinformatics/btp536. 
1400 cell proliferation', Developmental Biology, 304(1), pp. 34-45. doi: 10.1016/j.ydbio.2006.12.013.

Van Den Brink, S. C. et al. (2017) 'Single-cell sequencing reveals dissociation-induced gene expression in tissue subpopulations', Nature Methods, 14(10). doi: 10.1038/nmeth.4437.

Cannoodt, R., Saelens, W. and Saeys, Y. (2016) 'Computational methods for trajectory inference from single-cell transcriptomics', European Journal of Immunology, 46(11), pp. 24962506. doi: 10.1002/eji.201646347.

Carbon, S. et al. (2009) 'AmiGO: online access to ontology and annotation data', Bioinformatics. Oxford University Press, 25(2), pp. 288-289. doi: 10.1093/bioinformatics/btn615.

Chakraborty, P. et al. (2014) 'LIN28A Marks the Spermatogonial Progenitor Population and Regulates Its Cyclic Expansion', STEM CELLS, 32(4), pp. 860-873. doi: 10.1002/stem.1584.

Chen, E. Y. et al. (2013) 'Enrichr: interactive and collaborative HTML5 gene list enrichment analysis tool', BMC Bioinformatics, 14(1), p. 128. doi: 10.1186/1471-2105-14-128.

Chen, H. H. et al. (2014) 'DAZL limits pluripotency, differentiation, and apoptosis in developing primordial germ cells', Stem Cell Reports, 3(5), pp. 892-904. doi: 10.1016/j.stemcr.2014.09.003.

Chen, Y. et al. (2018) 'Single-cell RNA-seq uncovers dynamic processes and critical regulators in mouse spermatogenesis', Cell Research, 28(9), pp. 879-896. doi: 10.1038/s41422-018-0074y.

Choi, I. et al. (2003) 'Identification and characterization of ADAM32 with testis-predominant gene expression', Gene, 304(1-2), pp. 151-162. doi: 10.1016/S0378-1119(02)01202-7.

da Cruz, I. et al. (2016) 'Transcriptome analysis of highly purified mouse spermatogenic cell populations: gene expression signatures switch from meiotic-to post-meiotic-related processes at pachytene stage.', BMC genomics, 17(1), p. 294. doi: 10.1186/s12864-016-2618-1.

Engreitz, J. M. et al. (2013) 'The Xist IncRNA Exploits Three-Dimensional Genome Architecture to Spread Across the X Chromosome', Science, 341(6147), pp. 1237973-1237973. doi: 10.1126/science.1237973.

Ernst, C. et al. (2019) 'Staged developmental mapping and X chromosome transcriptional dynamics during mouse spermatogenesis', Nature Communications, 10, p. 1251. doi: 10.1038/s41467-019-09182-1.

Fallahi, M. et al. (2010) 'A Global Expression Switch Marks Pachytene Initiation during Mouse Male Meiosis', Genes, 1(3), pp. 469-483. doi: 10.3390/genes1030469.

Feng, X. H., Lin, X. and Derynck, R. (2000) 'Smad2, Smad3 and Smad4 cooperate with Sp1 to induce p15(Ink4B) transcription in response to TGF-beta.', The EMBO journal, 19(19), pp. 517893. doi: 10.1093/emboj/19.19.5178.

Fernandez, P. C. (2003) 'Genomic targets of the human c-Myc protein', Genes \& Development, 17(9), pp. 1115-1129. doi: 10.1101/gad.1067003.

Giorgetti, L. et al. (2016) 'Structural organization of the inactive X chromosome in the mouse.', Nature, in press(7613), pp. 1-5. doi: 10.1038/nature18589.

Goldberg, E. et al. (2010) 'LDHC: The Ultimate Testis-Specific Gene', Journal of Andrology, 31(1), pp. 86-94. doi: 10.2164/jandrol.109.008367.

Goymer, P. (2005) 'Which mean do you mean?', Nature genetics, 6(December), pp. 2005-2005. doi: $10.1080 / 0020739860170303$.

Green, C. D. et al. (2018) 'A Comprehensive Roadmap of Murine Spermatogenesis Defined by 
Single-Cell RNA-Seq', Developmental Cell, 46(5), p. 651-667.e10. doi: 10.1016/j.devcel.2018.07.025.

Griswold, M. D. (2016) 'Spermatogenesis: The Commitment to Meiosis', Physiological Reviews, 96(1), pp. 1-17. doi: 10.1152/physrev.00013.2015.

Grün, D. et al. (2015) 'Single-cell messenger RNA sequencing reveals rare intestinal cell types', Nature. Nature Research, 525(7568), pp. 251-255. doi: 10.1038/nature14966.

1448 Grün, D. et al. (2016) 'De Novo Prediction of Stem Cell Identity using Single-Cell Transcriptome Data', Cell Stem Cell, 19(2), pp. 266-277. doi: 10.1016/j.stem.2016.05.010.

Grün, D., Kester, L. and van Oudenaarden, A. (2014) 'Validation of noise models for single-cell transcriptomics.', Nature methods, 11(6), pp. 637-40. doi: 10.1038/nmeth.2930.

Grun, D. and van Oudenaarden, A. (2015) 'Design and Analysis of Single-Cell Sequencing Experiments.', Cell, 163(4), pp. 799-810. doi: 10.1016/j.cell.2015.10.039.

Hart, P. E. et al. (1999) 'Testis-Specific Murine Centrin, Cetn1: Genomic Characterization and Evidence for Retroposition of a Gene Encoding a Centrosome Protein', Genomics, 60(2), pp. 111-120. doi: 10.1006/geno.1999.5880.

Hasegawa, K. et al. (2015) 'SCML2 Establishes the Male Germline Epigenome through 10.1016/j.devcel.2015.01.014.

Hashimshony, T. et al. (2016) 'CEL-Seq2: sensitive highly-multiplexed single-cell RNA-Seq', Genome Biology. BioMed Central, 17(1), p. 77. doi: 10.1186/s13059-016-0938-8. Association. Taylor \& Francis, 84(406), pp. 502-516. doi: 10.1080/01621459.1989.10478797.

van der Heijden, G. W. et al. (2007) 'Chromosome-wide nucleosome replacement and H3.3 pp. 251-258. doi: 10.1038/ng1949.

Henderson, S. A. (1963) 'Differential Ribonucleic Acid Synthesis Of X And Autosomes During Meiosis.', Nature, 200, p. 1235. doi: 14089938.

Hermann, B. P. et al. (2018) 'The Mammalian Spermatogenesis Single-Cell Transcriptome, from Spermatogonial Stem Cells to Spermatids', Cell Reports, 25(6), p. 1650-1667.e8. doi: 10.1016/j.celrep.2018.10.026.

Jan, S. Z. et al. (2017) 'Unraveling transcriptome dynamics in human spermatogenesis', Development, p. dev.152413. doi: 10.1242/dev.152413.

Ji, Z. and Ji, H. (2016) 'TSCAN: Pseudo-time reconstruction and evaluation in single-cell RNAseq analysis', Nucleic Acids Research, 44(13), p. e117. doi: 10.1093/nar/gkw430.

Jiang, L. et al. (2017) 'RPL10L Is Required for Male Meiotic Division by Compensating for RPL10 during Meiotic Sex Chromosome Inactivation in Mice', Current Biology, 27(10), p. 14981505.e6. doi: 10.1016/j.cub.2017.04.017.

1483 Kasahara, T. et al. (2010) 'Genetic variation of melatonin productivity in laboratory mice under 
domestication', Proceedings of the National Academy of Sciences, 107(14), pp. 6412-6417. doi: 10.1073/pnas.0914399107.

Khalil, A. M., Boyar, F. Z. and Driscoll, D. J. (2004) 'Dynamic histone modifications mark sex chromosome inactivation and reactivation during mammalian spermatogenesis', Proc Natl Acad Sci U S A, 101(47), pp. 16583-16587. doi: 10.1073/pnas.0406325101.

Khil, P. P. et al. (2004) 'The mouse X chromosome is enriched for sex-biased genes not subject to selection by meiotic sex chromosome inactivation', Nature Genetics, 36(6), pp. 642-646. doi: 1491 10.1038/ng1368.

Kim, B., Cooke, H. J. and Rhee, K. (2012) 'DAZL is essential for stress granule formation implicated in germ cell survival upon heat stress', Development, 139, pp. 568-578. doi: 10.1242/dev.075846.

Koslowski, M. et al. (2006) 'The human X chromosome is enriched for germline genes expressed in premeiotic germ cells of both sexes', Human Molecular Genetics, 15(15), pp. 2392-2399. doi: 10.1093/hmg/ddl163.

Kuleshov, M. V. et al. (2016) 'Enrichr: a comprehensive gene set enrichment analysis web server 2016 update', Nucleic acids research, 44(W1), pp. W90-W97. doi: 10.1093/nar/gkw377.

Li, H. (2013) 'Aligning sequence reads, clone sequences and assembly contigs with BWA-MEM'.

Lukassen, S. et al. (2018) 'Characterization of germ cell differentiation in the male mouse through single-cell RNA sequencing', Scientific Reports, 8(1), p. 6521. doi: 10.1038/s41598-01824725-0.

Luo, J., Megee, S. and Dobrinski, I. (2009) 'Asymmetric Distribution of UCH-L1 in

Luo, M. et al. (2013) 'MEIOB exhibits single-stranded DNA-binding and exonuclease activities and is essential for meiotic recombination.', Nature communications, 4(1), p. 2788. doi: 10.1038/ncomms3788.

Luo, M. et al. (2015) 'Polycomb protein SCML2 associates with USP7 and counteracts histone $\mathrm{H} 2 \mathrm{~A}$ ubiquitination in the XY chromatin during male meiosis.', PLoS genetics. Edited by J. C. Schimenti. Public Library of Science, 11(1), p. e1004954. doi: 10.1371/journal.pgen.1004954.

Mahadevaiah, S. K. et al. (2001) 'Recombinational DNA double-strand breaks in mice precede synapsis.', Nature genetics. Nature Publishing Group, 27(3), pp. 271-6. doi: 10.1038/85830.

Mao, Q. et al. (2016) 'Principal Graph and Structure Learning Based on Reversed Graph Embedding', IEEE Transactions on Pattern Analysis and Machine Intelligence, XX(X), pp. 1-1. doi: 10.1109/TPAMI.2016.2635657.

Margolin, G. et al. (2014) 'Integrated transcriptome analysis of mouse spermatogenesis', BMC Genomics, 15(1), p. 39. doi: 10.1186/1471-2164-15-39.

Matson, C. K. et al. (2010) 'The Mammalian Doublesex Homolog DMRT1 Is a Transcriptional Gatekeeper that Controls the Mitosis versus Meiosis Decision in Male Germ Cells', Developmental Cell, 19(4), pp. 612-624. doi: 10.1016/j.devcel.2010.09.010.

Matys, V. (2006) 'TRANSFAC(R) and its module TRANSCompel(R): transcriptional gene regulation in eukaryotes', Nucleic Acids Research, 34(90001), pp. D108-D110. doi: 10.1093/nar/gkj143. 
Meistrich, M. L. and Hess, R. A. (2013) 'Assessment of Spermatogenesis Through Staging of Seminiferous Tubules', in Carrell, D. T. and Aston, K. I. (eds) Spermatogenesis: Methods and Protocols. Totowa, NJ: Humana Press, pp. 299-307. doi: 10.1007/978-1-62703-038-0_27.

Mering, C. v. (2003) 'STRING: a database of predicted functional associations between proteins', Nucleic Acids Research. Oxford University Press, 31(1), pp. 258-261. doi: 10.1093/nar/gkg034.

Monesi, V. (1965) 'Differential rate of ribonucleic acid synthesis in the autosomes and sex chromosomes during male meiosis in the mouse', Chromosoma, 17(1), pp. 11-21. doi: 10.1007/BF00285153.

Muraro, M. J. et al. (2016) 'A Single-Cell Transcriptome Atlas of the Human Pancreas', Cell Systems, 3(4), p. 385-394.e3. doi: 10.1016/j.cels.2016.09.002.

Nakata, H. et al. (2015) 'Quantitative analysis of the cellular composition in seminiferous tubules in normal and genetically modified infertile mice.', The journal of histochemistry and cytochemistry: official journal of the Histochemistry Society, 63(2), pp. 99-113. doi: 10.1369/0022155414562045.

Namekawa, S. H. et al. (2006) 'Post-meiotic Sex Chromatin in the Male Germline of Mice', Current Biology, 16(7), pp. 660-667. doi: 10.1016/j.cub.2006.01.066.

Nguyen, T. B. et al. (2002) 'Characterization and Expression of Mammalian Cyclin B3, a Prepachytene Meiotic Cyclin', Journal of Biological Chemistry, 277(44), pp. 41960-41969. doi: 10.1074/jbc.M203951200.

Nora, E. P. et al. (2012) 'Spatial partitioning of the regulatory landscape of the X-inactivation centre', Nature, 485(7398), pp. 381-385. doi: 10.1038/nature11049.

O'Shaughnessy, P. J., Morris, I. D. and Baker, P. J. (2008) 'Leydig cell re-generation and expression of cell signaling molecules in the germ cell-free testis', Reproduction, 135(6), pp. 851-858. doi: 10.1530/REP-07-0529.

Page, J. et al. (2012) 'Inactivation or non-reactivation: what accounts better for the silence of sex chromosomes during mammalian male meiosis?', Chromosoma, 121(3), pp. 307-326. doi: 10.1007/s00412-012-0364-y.

Petropoulos, S. et al. (2016) 'Single-Cell RNA-Seq Reveals Lineage and X Chromosome Dynamics in Human Preimplantation Embryos.', Cell, 165(4), pp. 1012-26. doi: 10.1016/j.cell.2016.03.023.

Portales-Casamar, E. et al. (2010) 'JASPAR 2010: the greatly expanded open-access database of transcription factor binding profiles.', Nucleic acids research. Oxford University Press, 38(Database issue), pp. D105-10. doi: 10.1093/nar/gkp950.

Primakoff, P. and Myles, D. G. (2000) 'The ADAM gene family: Surface proteins with adhesion and protease activity', Trends in Genetics, 16(2), pp. 83-87. doi: 10.1016/S01689525(99)01926-5.

Qiu, X. et al. (2017) 'Reversed graph embedding resolves complex single-cell trajectories.', Nature methods, 14(10), pp. 979-982. doi: 10.1038/nmeth.4402.

Raivo, K. (2013) pheatmap: Pretty Heatmaps.

Royo, H. et al. (2015) 'Silencing of X-Linked MicroRNAs by Meiotic Sex Chromosome Inactivation', PLoS Genetics, 11(10), pp. 1-15. doi: 10.1371/journal.pgen.1005461.

Russell, L. D. et al. (1993) Histological and Histopathological Evaluation of the Testis. doi: 10.1111/j.1365-2605.1993.tb01156.x. 
Shin, Y.-H. et al. (2010) 'Hormad1 mutation disrupts synaptonemal complex formation, recombination, and chromosome segregation in mammalian meiosis.', PLoS genetics. Edited by R. S. Hawley, 6(11), p. e1001190. doi: 10.1371/journal.pgen.1001190.

Shiu, P. K. T. et al. (2001) 'Meiotic Silencing by Unpaired DNA', Cell, 107(7), pp. 905-916. doi: 10.1016/S0092-8674(01)00609-2.

Sun, F. et al. (2015) 'Nuclear localization of PRDM9 and its role in meiotic chromatin modifications and homologous synapsis.', Chromosoma, 124(3), pp. 397-415. doi: $10.1007 / \mathrm{s} 00412-015-0511-3$.

Supek, F. et al. (2011) 'Revigo summarizes and visualizes long lists of gene ontology terms', PLoS ONE. Edited by C. Gibas, 6(7), p. e21800. doi: 10.1371/journal.pone.0021800.

Szklarczyk, D. et al. (2017) 'The STRING database in 2017: quality-controlled protein-protein association networks, made broadly accessible', Nucleic Acids Research. Oxford University Press, 45(D1), pp. D362-D368. doi: 10.1093/nar/gkw937.

Tibshirani, R., Walther, G. and Hastie, T. (2001) 'Estimating the number of clusters in a data set via the gap statistic', Journal of the Royal Statistical Society: Series B (Statistical Methodology), 63(2), pp. 411-423. doi: 10.1111/1467-9868.00293.

Turinetto, V. and Giachino, C. (2015) 'Multiple facets of histone variant H2AX: a DNA doublestrand-break marker with several biological functions.', Nucleic acids research, 43(5), pp. 248998. doi: 10.1093/nar/gkv061.

Turner, J. M. A. et al. (2004) 'BRCA1, Histone H2AX Phosphorylation, and Male Meiotic Sex Chromosome Inactivation', Current Biology, 14(23), pp. 2135-2142. doi: 10.1016/j.cub.2004.11.032.

Turner, J. M. A. (2015) 'Meiotic Silencing in Mammals.', Annual review of genetics, 49(1), pp. 395-412. doi: 10.1146/annurev-genet-112414-055145.

Vandesompele, J. et al. (2002) 'Accurate normalization of real-time quantitative RT-PCR data by geometric averaging of multiple internal control genes', Genome Biology, 3(7), p. research0034.1. doi: 10.1186/gb-2002-3-7-research0034.

Ventela, S. et al. (2000) 'Expression of green fluorescent protein under beta-actin promoter in living spermatogenic cells of the mouse: stage-specific regulation by FSH', International Journal of Andrology, 23(4), pp. 236-242. doi: 10.1046/j.1365-2605.2000.00237.x.

Vertesy, A. (2017) 'MarkdownReports: An R function library to create scientific figures and markdown reports easily. Update v2.9.5'. doi: 10.5281/zenodo.1014353.

Wang, J. et al. (2017) 'NRF1 coordinates with DNA methylation to regulate spermatogenesis', The FASEB Journal, 31(11), pp. 4959-4970. doi: 10.1096/fj.201700093R.

Xue, H. et al. (1999) 'HemT-3, an alternative transcript of mouse gene HemT specific to male germ cells.', Gene, 240(1), pp. 193-9. doi: 10.1016/S0378-1119(99)00401-1.

Yoshinaga, K. et al. (1991) 'Role of c-kit in mouse spermatogenesis: identification of spermatogonia as a specific site of c-kit expression and function.', Development (Cambridge, England), 113(2), pp. 689-99.

Zimmermann, C. et al. (2015) 'Research Resource: The Dynamic Transcriptional Profile of Sertoli Cells During the Progression of Spermatogenesis', Molecular Endocrinology, 29(4), pp. 627-642. doi: 10.1210/me.2014-1356. 
A

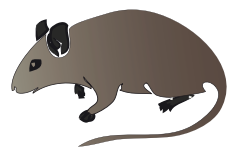

Dissection of testes of DAZL-GFP mouse (1)

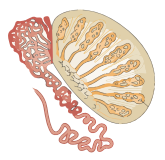

$D A Z L-G F P+$ Spermatogonia \& Prophase I spermatocytes found at the basal layers
Dissociation of seminiferous tubules into single cell (2)
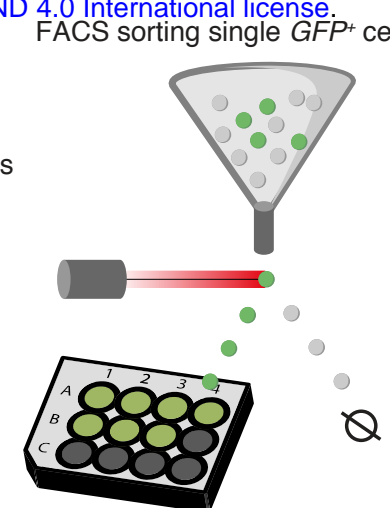

CEL-Seq2 single-cell prep. \& sequencing (4) in vivo validation by single-molecule RNA FISH (7)

B
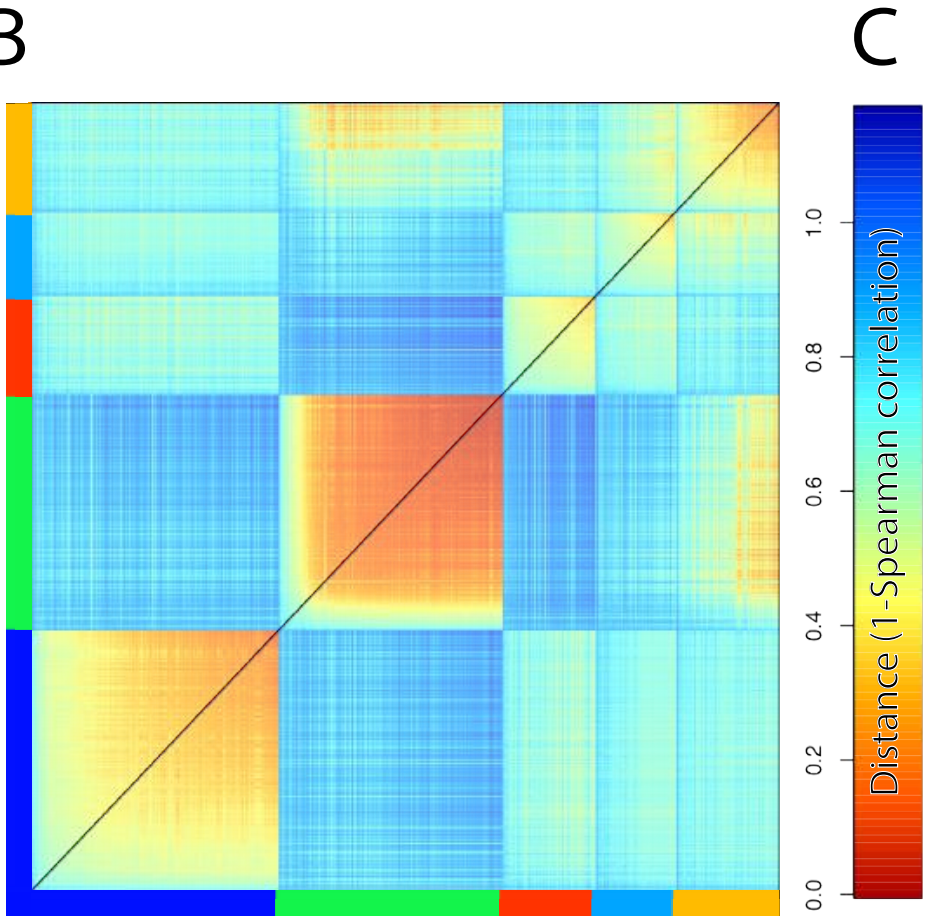

Clusters
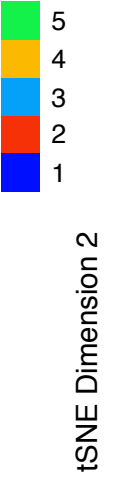

tSNE Dimension 1

D

Stra6

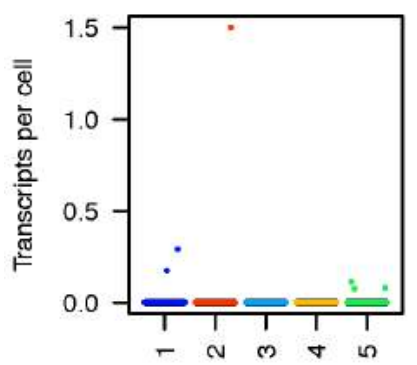

Sertoli cell specific

Kit

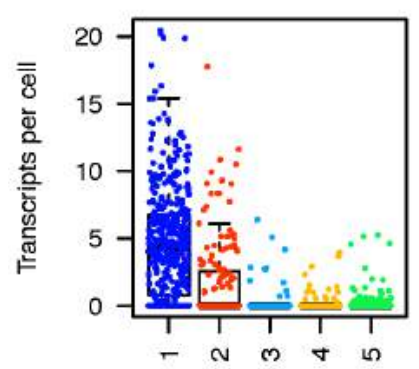

Differentiating spermatogonia
Amh

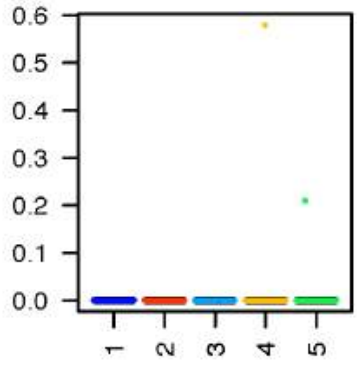

Leydig cell specific

Stra8

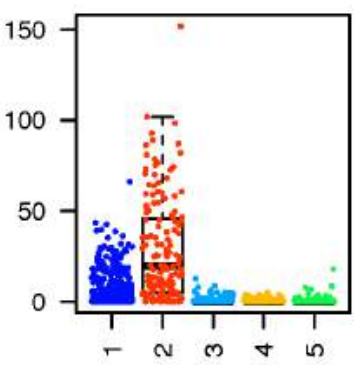

Meiotic inducer

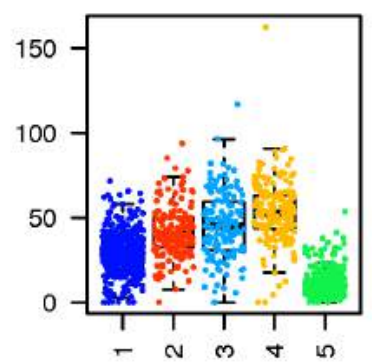

Spermatogonia \& prophase specific

Ccnb3

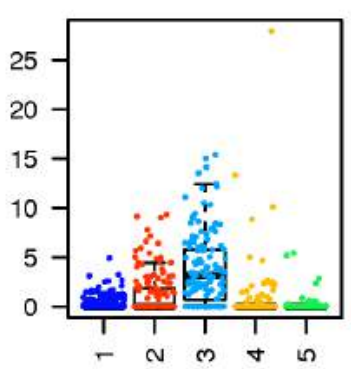

Meiotic cyclin on chr. X

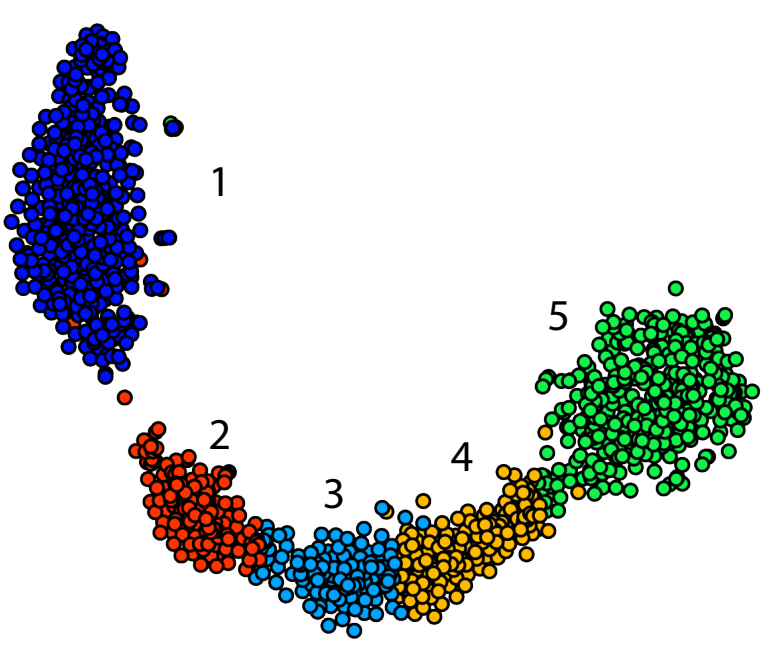

emporal reconstruction \& gene expression waves (6)
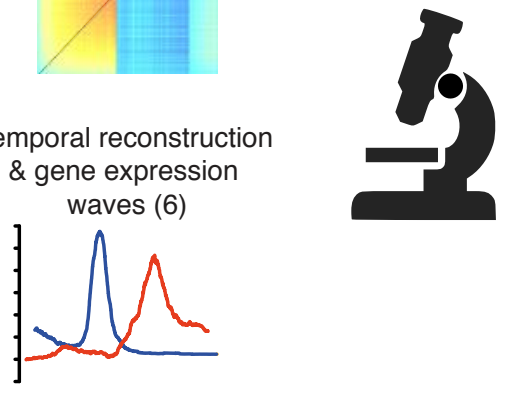
\& clustering (5)

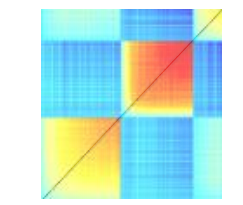

\section{$x^{2}$}


A

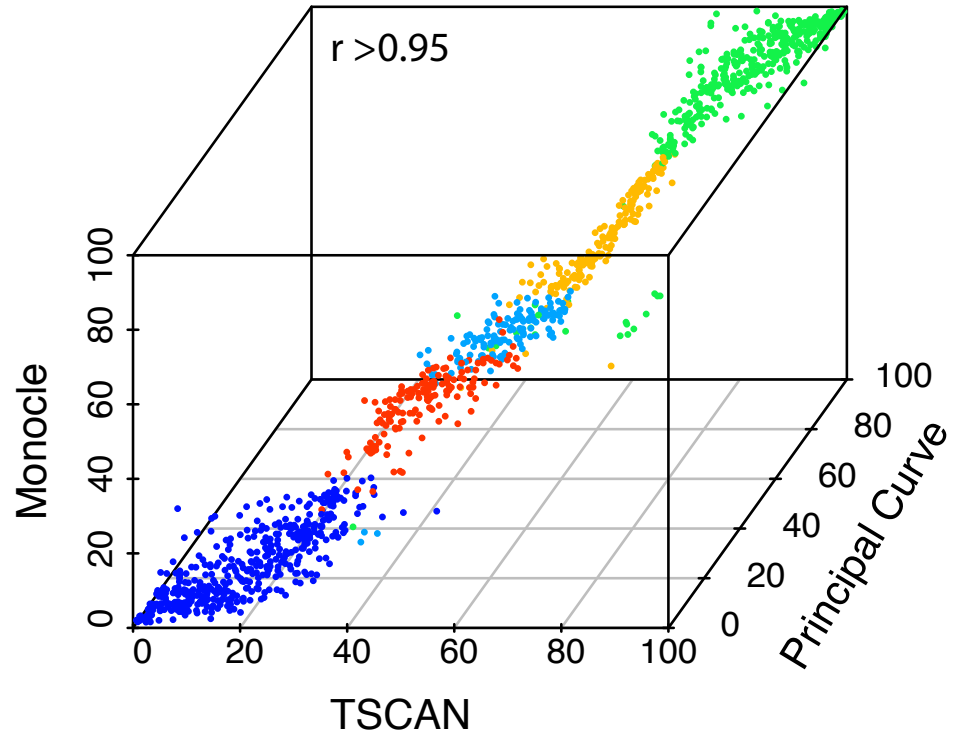

$B^{\text {ernational license. }}$

under aCC-BY-ND 4.0 B
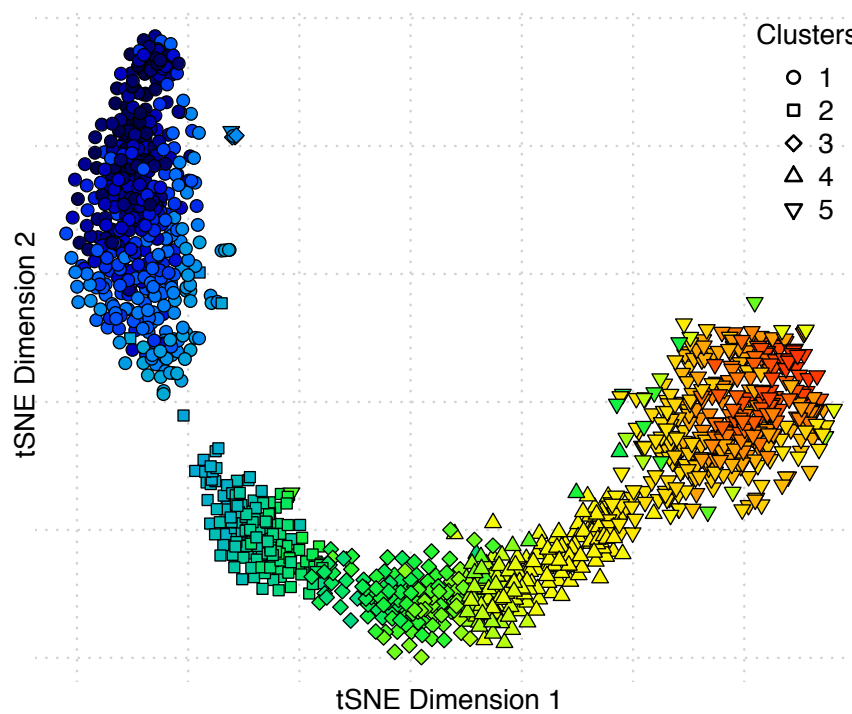

100

80

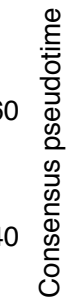

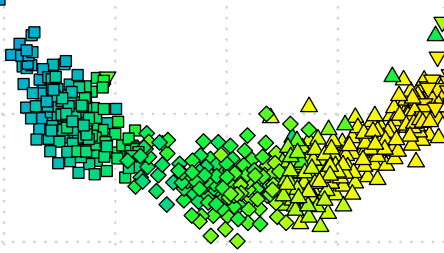

tSNE Dimension 1

Pseudotime is scaled $0-100$ for all algorithms.

C

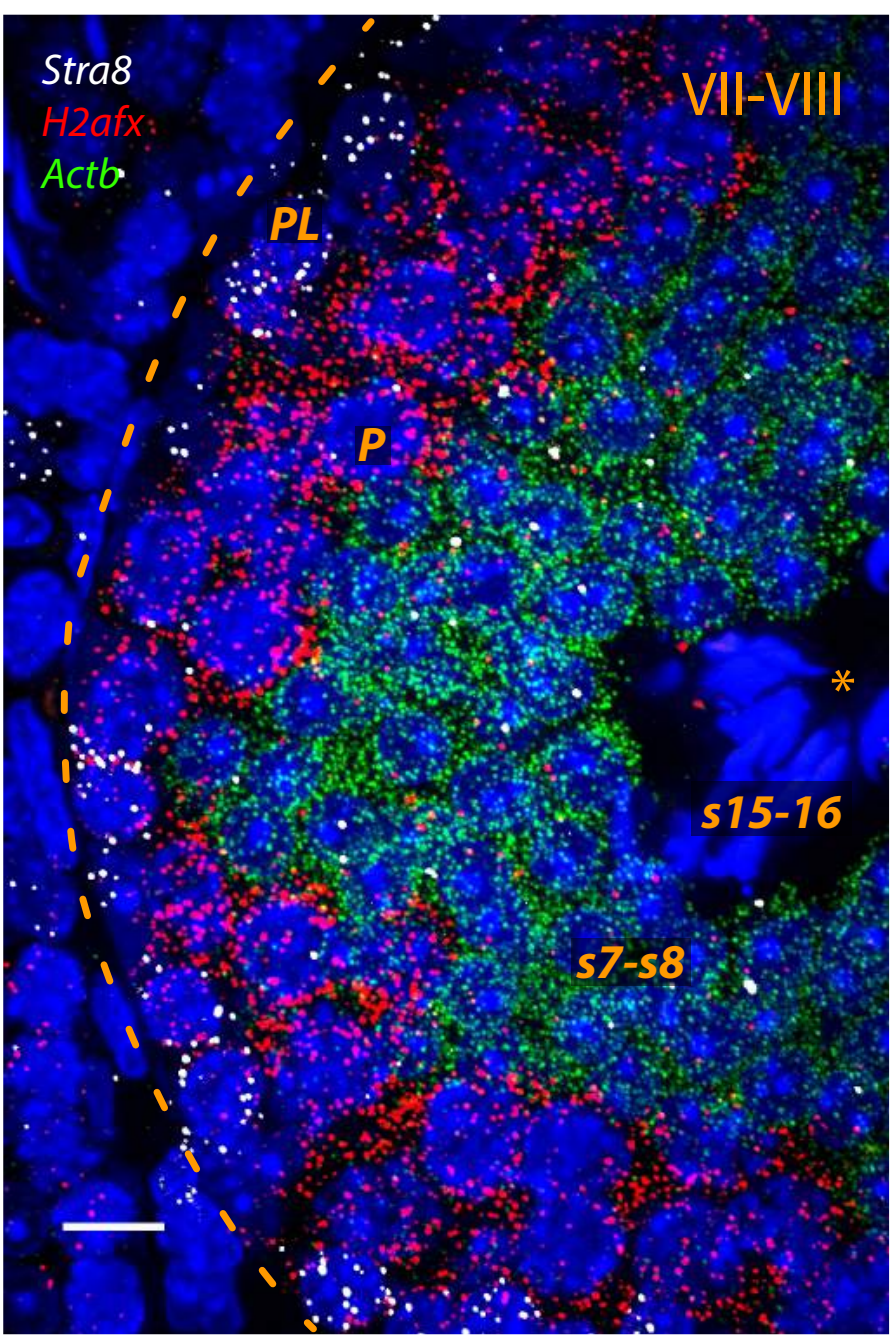

D

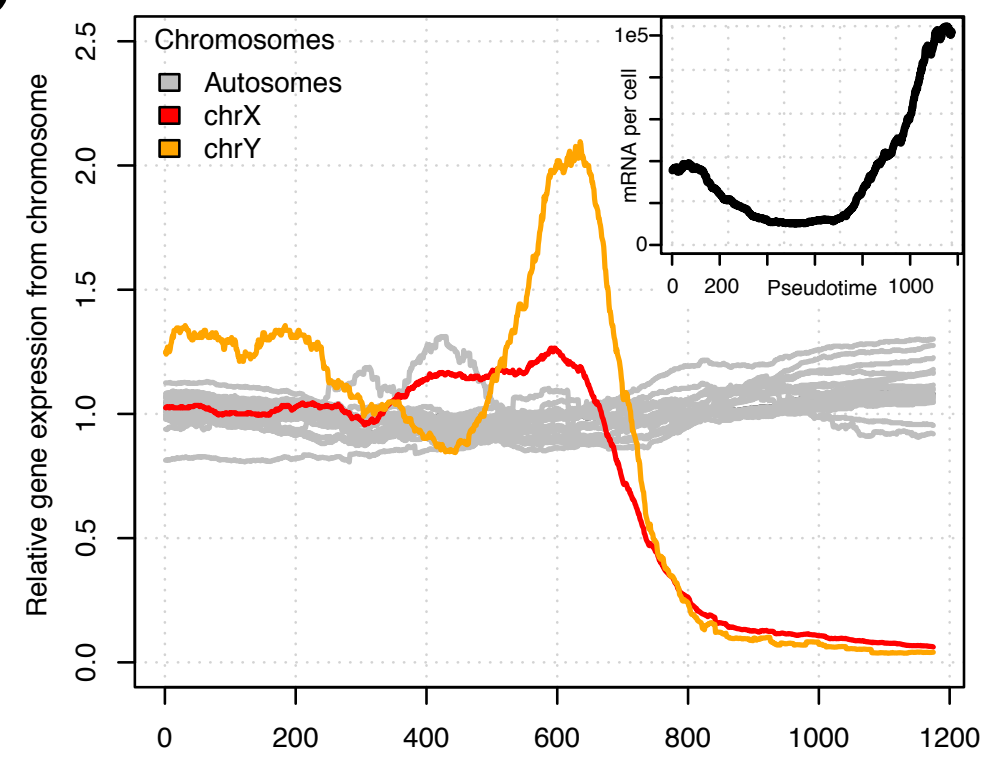

E

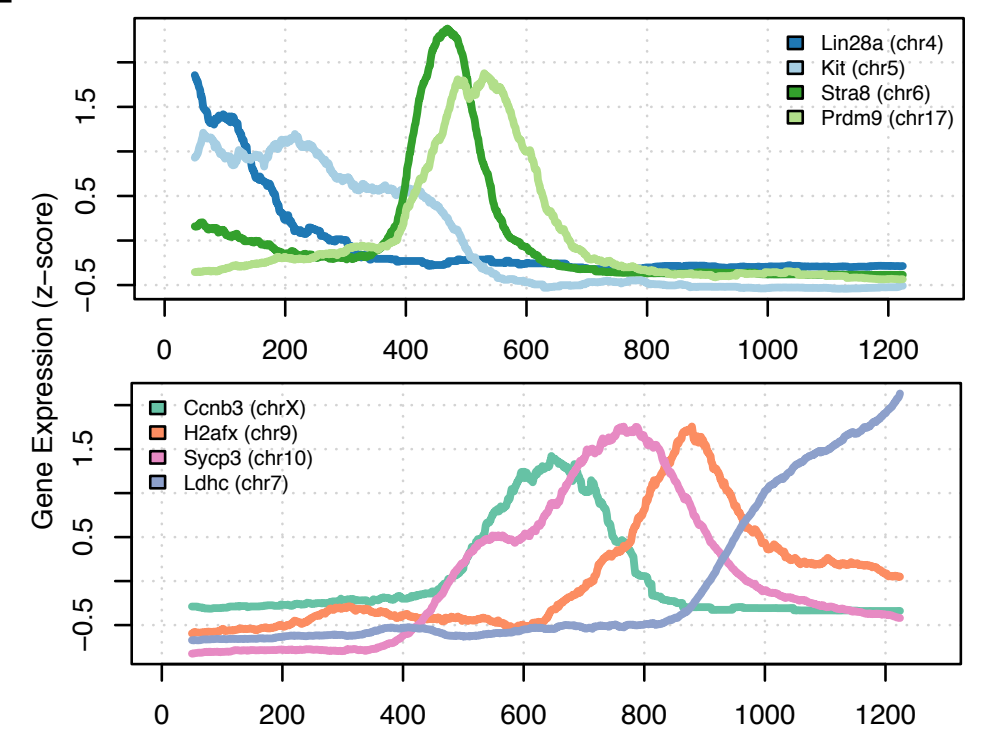

Pseudotime 
A
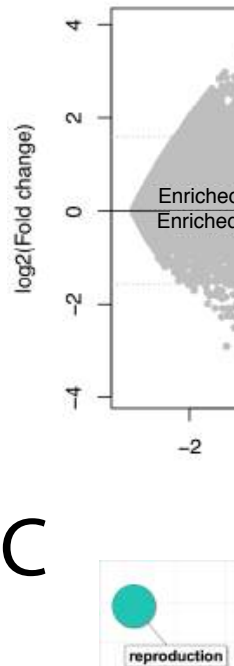

-

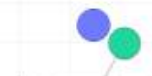

DNA recombination

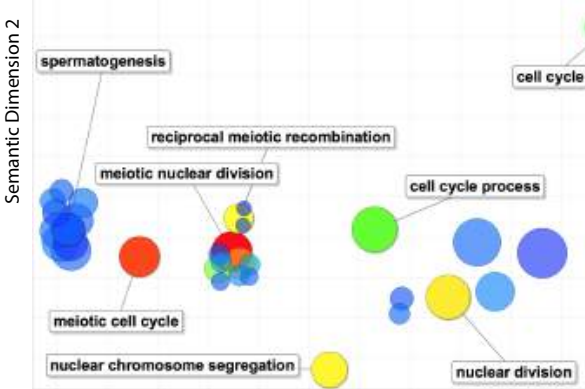

D

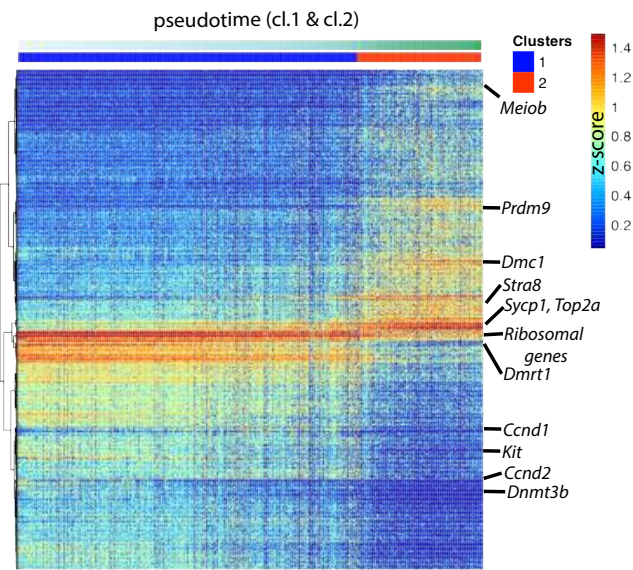

B

4-fold DEG

spermatogonia

mitotic cell cycle cell cycle
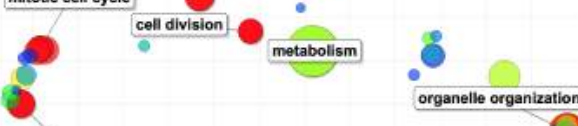

regulation of cell cycie

chromosome organization

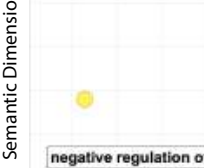

(1)

negative regulation of gene expression

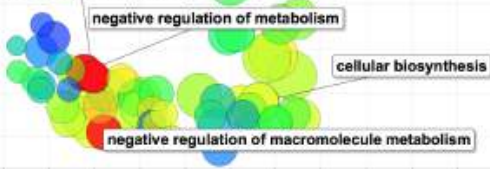

Semantic Dimension 1

\begin{tabular}{lrrrr} 
Name & P-value & $\begin{array}{r}\text { Adjusted } \\
\text { p-value }\end{array}$ & Z-score & $\begin{array}{r}\text { Combined } \\
\text { score }\end{array}$ \\
\hline SP1 (mouse) & $4.333 \mathrm{e}-12$ & $1.304 \mathrm{e}-9$ & -1.64 & 42.90 \\
SMADA (mouse) & $7.119 \mathrm{e}-9$ & $7.143 \mathrm{e}-7$ & -1.66 & 31.08 \\
EGR1 (mouse) & $2.0996-7$ & 0.00001203 & -1.65 & 25.47 \\
Myb (mouse) & 0.00002205 & 0.0004741 & -1.54 & 16.51 \\
MYC (mouse) & 0.00003779 & 0.0007129 & -1.50 & 15.26 \\
ELK1 (mouse) & 0.00005966 & 0.0009452 & -1.42 & 13.85 \\
TP53 (mouse) & 0.0001232 & 0.001685 & -1.48 & 13.28 \\
NR51 (mouse) & 0.0003114 & 0.003905 & -1.37 & 11.10
\end{tabular}

E

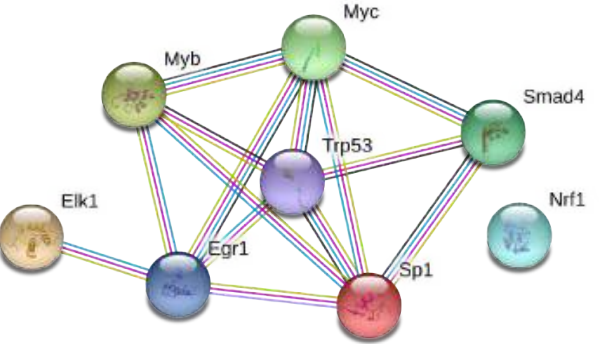

Interactions

/ curated databases

/ experimental data

/ textmining

/ co-expression

/ homology
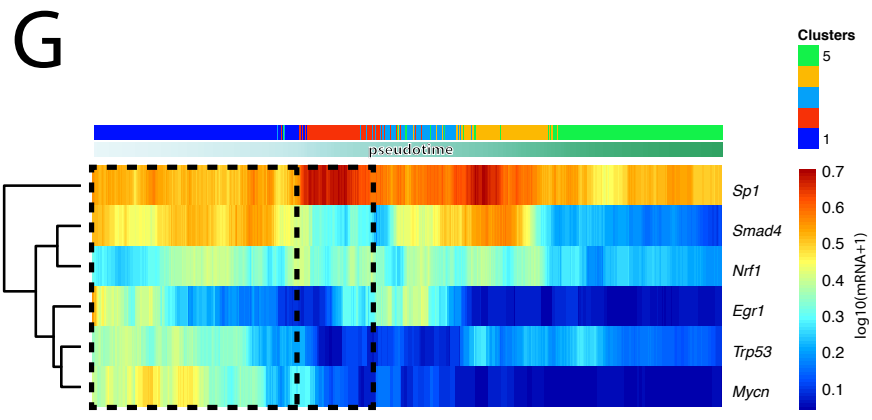
bioRxiv preprint doi: https://doi.org/10.1101/665372; this version posted July 8,2019 . The copyright holder for this preprint (which was not
certified by peer review) is the author/funder, who has granted bioRxiv a license to display the preprint in perpetuity. It is made available
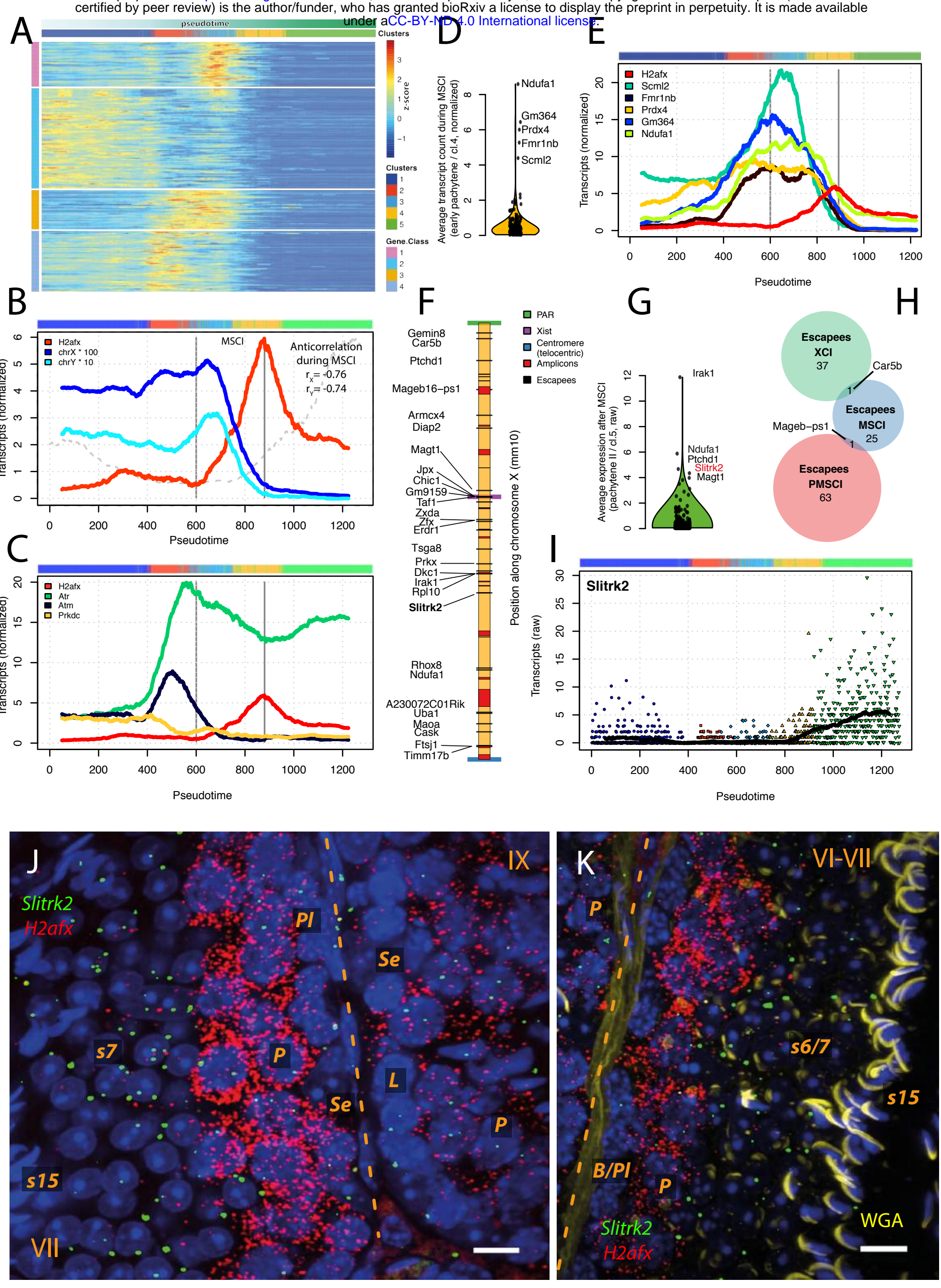
(isplay the preprint in perpetuity. It is made available under ACC-BY-ND 4.0 International license.

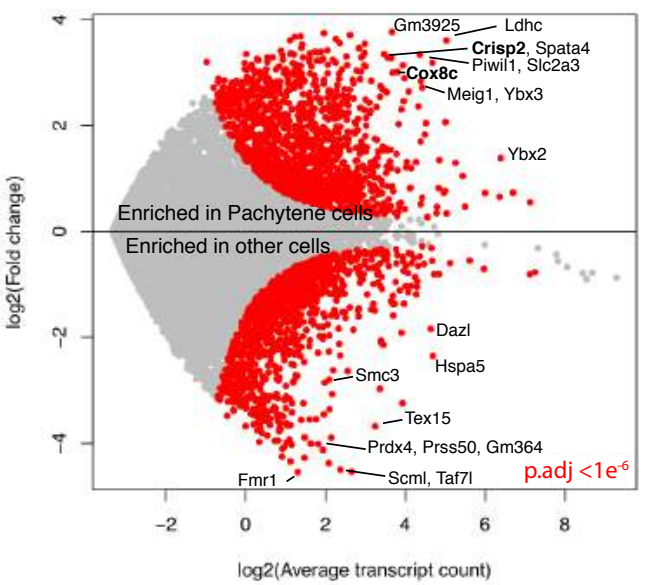

D

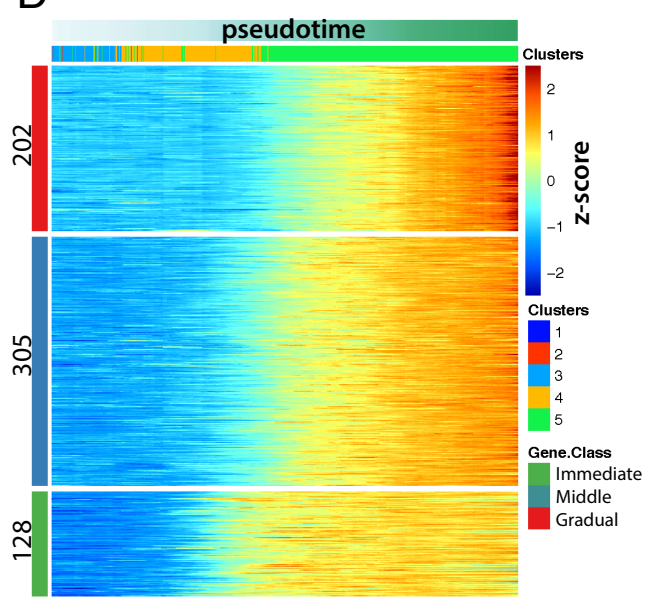

G

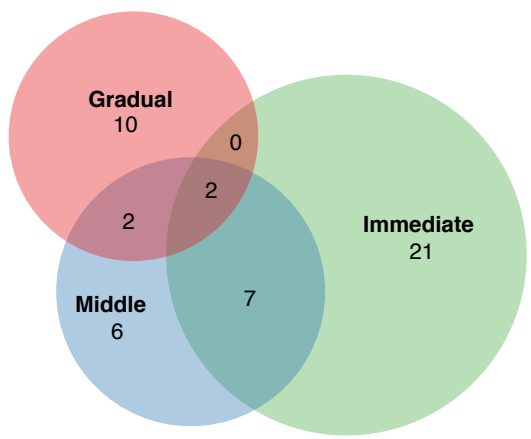

GO-terms Cellular Component and Biological Process, FDR $<0.001$

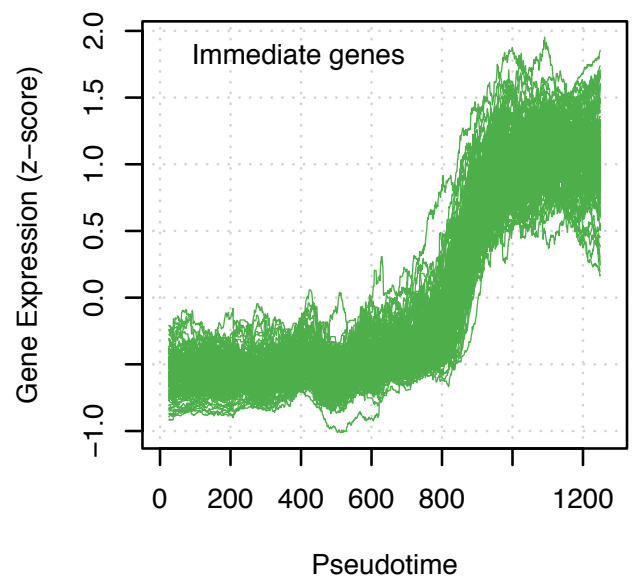

E

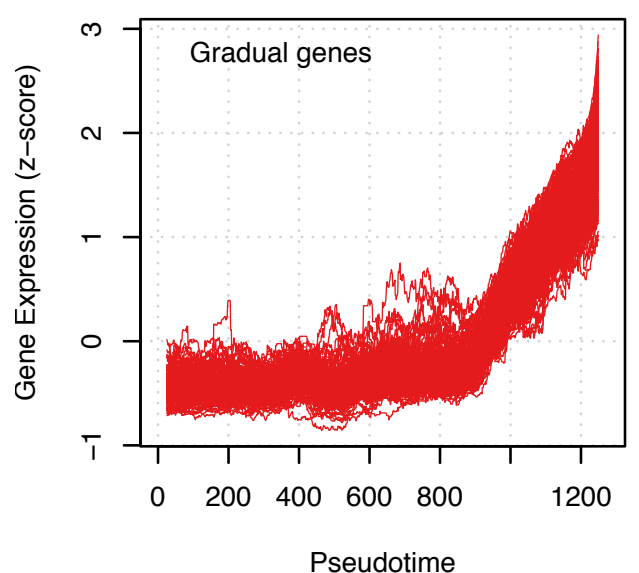

$\mathrm{H}$

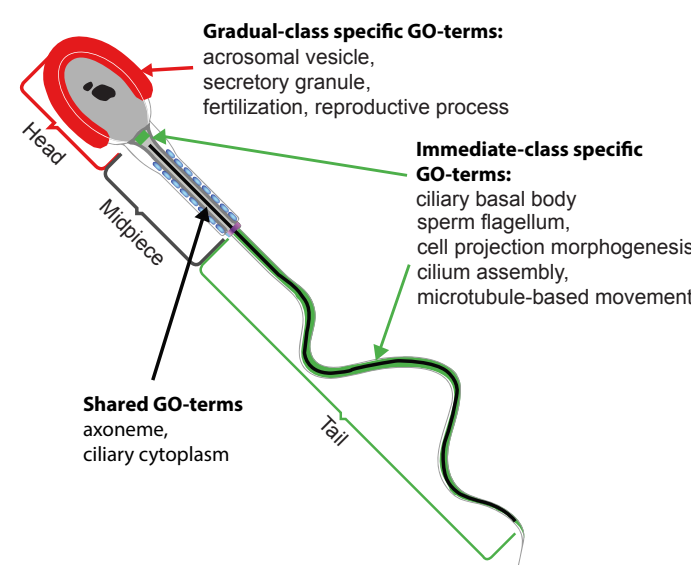

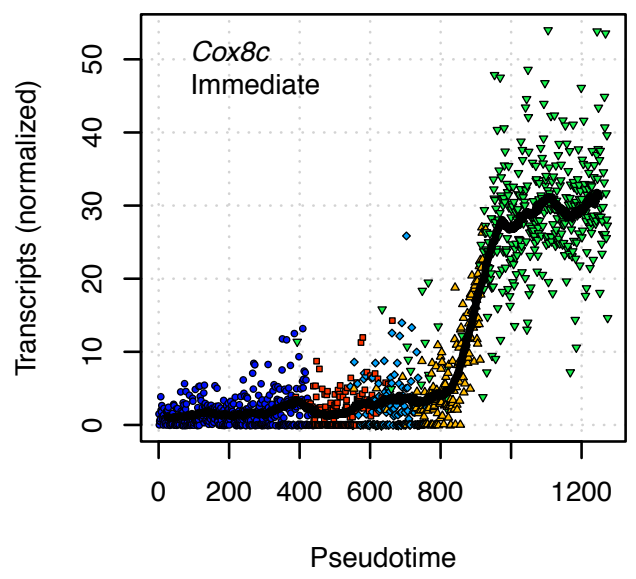

$\mathrm{F}$
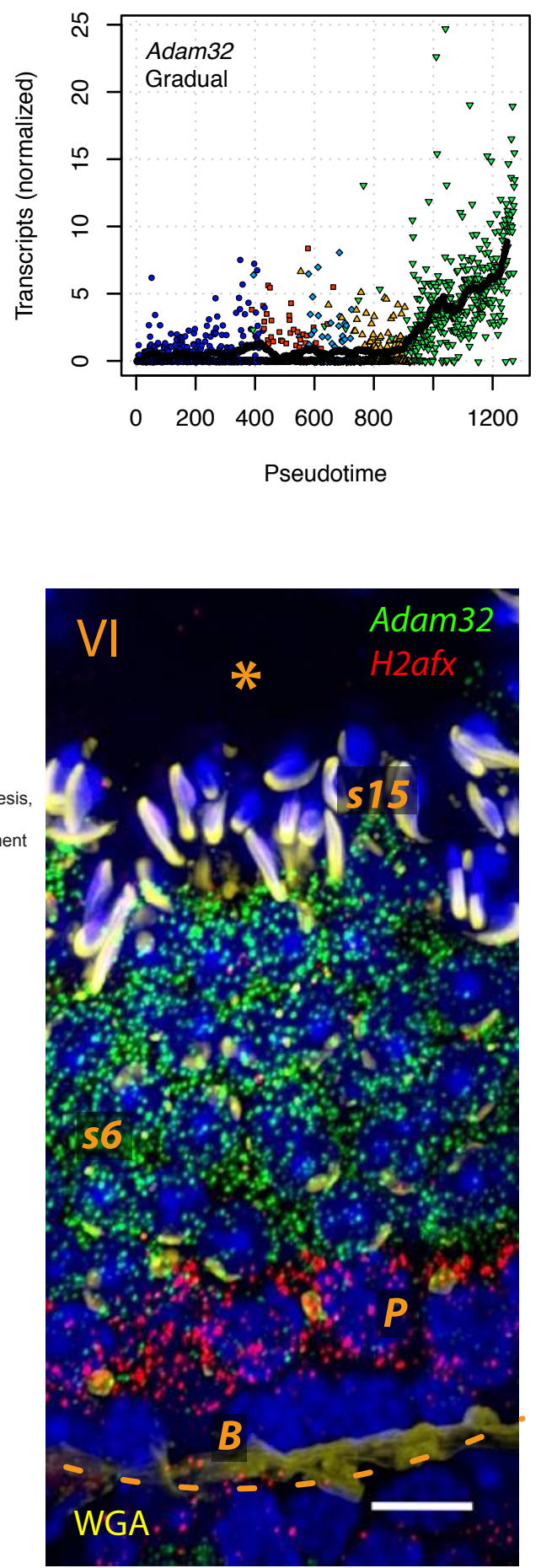